\title{
Covalent and Noncovalent Conjugation of Degradable Polymer Nanoparticles to T Lymphocytes
}

\author{
Tanja Thomsen, Regina Reissmann, Elisa Kaba, Britta Engelhardt, and Harm-Anton Klok*
}

Cite This: Biomacromolecules 2021, 22, 3416-3430

Read Online
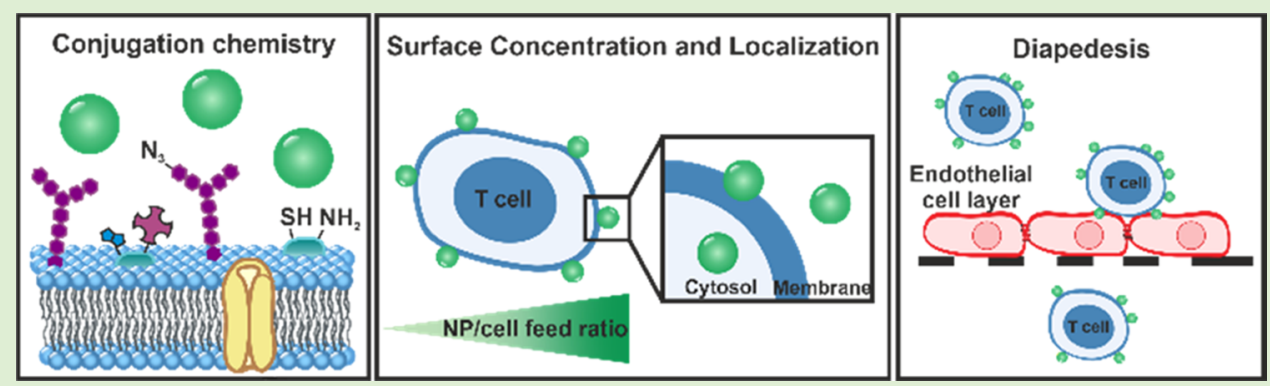

ABSTRACT: Cells are attractive as carriers that can help to enhance control over the biodistribution of polymer nanomedicines. One strategy to use cells as carriers is based on the cell surface immobilization of the nanoparticle cargo. While a range of strategies can be used to immobilize nanoparticles on cell surfaces, only limited effort has been made to investigate the effect of these surface modification chemistries on cell viability and functional properties. This study has explored seven different approaches for the immobilization of poly(lactic acid) (PLA) nanoparticles on the surface of two different T lymphocyte cell lines. The cell lines used were human Jurkat $\mathrm{T}$ cells and $\mathrm{CD}^{+} \mathrm{T}_{\mathrm{EM}}$ cells. The latter cells possess blood-brain barrier (BBB) migratory properties and are attractive for the development of cell-based delivery systems to the central nervous system (CNS). PLA nanoparticles were immobilized either via covalent active ester-amine, azide-alkyne cycloaddition, and thiol-maleimide coupling, or via noncovalent approaches that use lectin-carbohydrate, electrostatic, or biotin-NeutrAvidin interactions. The cell surface immobilization of the nanoparticles was monitored with flow cytometry and confocal microscopy. By tuning the initial nanoparticle/cell ratio, $\mathrm{T}$ cells can be decorated with up to $\sim 185$ nanoparticles/cell as determined by confocal microscopy. The functional properties of the nanoparticle-decorated cells were assessed by evaluating their binding to ICAM-1, a key protein involved in the adhesion of CD4 ${ }^{+}$ $\mathrm{T}_{\mathrm{EM}}$ cells to the $\mathrm{BBB}$ endothelium, as well as in a two-chamber model in vitro BBB migration assay. It was found that the migratory behavior of $\mathrm{CD}^{+} \mathrm{T}_{\mathrm{EM}}$ cells carrying carboxylic acid-, biotin-, or Wheat germ agglutinin (WGA)-functionalized nanoparticles was not affected by the presence of the nanoparticle payload. In contrast, however, for cells decorated with maleimide-functionalized nanoparticles, a reduction in the number of migratory cells compared to the nonmodified control cells was observed. Investigating and understanding the impact of nanoparticle-cell surface conjugation chemistries on the viability and properties of cells is important to further improve the design of cell-based nanoparticle delivery systems. The results of this study present a first step in this direction and provide first guidelines for the surface modification of $\mathrm{T}$ cells, in particular in view of their possible use for drug delivery to the CNS.

\section{INTRODUCTION}

The treatment of diseases with low-molecular-weight drugs often suffers from unfavorable pharmacokinetics, which restricts drug efficiency and can result in undesirable side effects to patients. To some extent, these drawbacks can be mitigated by administering drugs as nanomedicines, for example, in the form of nanosized particle formulations (micelles, polymersomes, solid core nanoparticles) or as polymer-drug conjugates. ${ }^{1-6}$ The use of polymers or polymer nanoparticle-based carriers can improve control over biodistribution and also provides opportunities for targeted delivery. In many instances, however, in particular in the context of cancer chemotherapy, only the minority fraction of these nanoparticles arrives at the desired destination while the majority ends up in off-target organs. ${ }^{7,8}$

Cell-based carriers are very promising to further enhance control over the biodistribution of therapeutic nanoparticles. $^{9-20}$ Noncovalent binding of polymer nanoparticles on the surface of red blood cells, for example, significantly

Received: April 16, 2021

Revised: June 15, 2021

Published: June 25, 2021 
increases the blood circulation time $\mathrm{e}^{21,22}$ and allows for enhanced accumulation in the lungs, while reducing uptake in liver and spleen. ${ }^{23-25}$ Another class of cells that has been used for the transport of therapeutic nanoparticles are $\mathrm{T}$ cells. $\mathrm{T}$ cells can possess tumor migratory properties and can penetrate tumor tissue, which makes these cells attractive vectors for the delivery of nanoparticles for cancer chemotherapy. $^{26-28}$

To use cells as carriers for the transport of nanoparticles, the cargo must be either internalized by the cells or attached to the cell surface. A plethora of strategies have been used to attach nanoparticles to cell surfaces. ${ }^{14,29-31}$ One approach involves the use of noncovalent interactions such as electrostatic adsorption, $^{24,32}$ lipophilic insertion into the cell membrane, ${ }^{33,34}$ and receptor-ligand interactions (carbohydratelectin, ${ }^{35,36}$ biotin-avidin, ${ }^{39,38}$ receptor-antibody ${ }^{39-41}$ ) to immobilize nanoparticles on cell surfaces. In addition to providing manifold opportunities to noncovalently attach nanoparticles, the cell membrane also presents a wide range of functional groups such as amine, thiol, hydroxyl, and carboxylic acid groups that can be used to covalently immobilize nanoparticles. Moreover, the use of metabolic glycoengineering strategies allows to introduce non-natural functional groups such as azido groups, which further expands the chemical diversity of the cell membrane and enables bioorthogonal covalent coupling reactions. ${ }^{42}$ Examples of covalent chemistries that have been used to attach nanoparticles to the cell surface include reaction of $N$-hydroxysulfosuccinimide (NHS)-ester activated nanoparticles with cell surface amino groups, or of dithiopyridyl- and maleimide-functionalized nanoparticles with free thiol groups available on the cell surface. $^{26,43-45}$ Surface saccharides of cancer cells have been explored to couple boronic acid-modified nanoparticles. ${ }^{46}$ Oxidation of the terminal alcohol groups of sialic acid residues generates aldehyde groups on the cell surface, which can be reacted with amine-functionalized nanoparticles to form a Schiff base linkage that can be further reduced to a stable secondary amine bond. ${ }^{47}$ Finally, triarylphosphine- or cyclooctyne-modified nanoparticles can be covalently attached to azido groups on the cell surface via Staudinger ligation or $\mathrm{Cu}$ free click chemistry. ${ }^{48,49}$

While there is ample evidence that demonstrates that cellmediated delivery is a powerful strategy to enhance control over the biodistribution of polymer nanoparticles, only limited effort has been made to explore and investigate the chemistry that underlies the surface modification of cell-based carriers with nanoparticle cargo. In spite of the availability of a plethora of conjugation strategies, most studies resort to a single type of conjugation chemistry. Investigating and understanding the impact of different nanoparticle-cell surface conjugation chemistries on the viability and functional properties of the cells could provide guidelines to further improve the design of cell-based nanoparticle delivery systems. This article presents the results of a comparative analysis that has evaluated seven different conjugation chemistries for the immobilization of poly(lactic acid) (PLA) nanoparticles on the surface of two different $\mathrm{T}$ lymphocyte cell lines. For this study, PLA nanoparticles were selected as they are widely used as biodegradable drug nanocarriers. ${ }^{5,50}$ As a first, model cell line, human Jurkat $\mathrm{T}$ cells were used. The second class of $\mathrm{T}$ cells used in this study are activated effector/memory $\mathrm{CD} 4^{+}$ helper SJL/PLP7 T cells ( $\mathrm{CD}^{+} \mathrm{T}_{\mathrm{EM}}$ cells). These cells possess blood-brain barrier (BBB) migratory properties and are attractive candidates for the development of cell-based drug delivery systems to the central nervous system (CNS). ${ }^{51}$ For each of the different chemistries, nanoparticle-cell surface conjugation was monitored by flow cytometry and the concentration and position of the nanoparticles on cell surface were quantitatively analyzed with confocal microscopy. Using flow cytometry, the impact of the different conjugation chemistries on the viability and functional properties of the $\mathrm{CD}^{+} \mathrm{T}_{\mathrm{EM}}$ cells was assessed and compared. In a final set of experiments, the influence of the nanoparticle cell surface modification on two key functional characteristics of the CD4 ${ }^{+}$ $\mathrm{T}_{\mathrm{EM}}$ cells was evaluated. A first experiment sought to probe the ability of nanoparticle-decorated cells to bind to ICAM-1, which has been identified as a critical endothelial adhesion molecule that mediates the interaction of $\mathrm{CD} 4^{+} \mathrm{T}_{\mathrm{EM}}$ cells with the $\mathrm{BBB}$. Finally, the BBB migratory properties of the cells were studied in a two-chamber setup using an in vitro $\mathrm{BBB}$ model.

\section{EXPERIMENTAL SECTION}

Methods. Particle Sizes and $\zeta$-Potentials. Particle sizes and $\zeta$ potentials were measured by dynamic light scattering (DLS) using a Zetasizer Nano ZS instrument (Malvern). Size measurements were performed at a nanoparticle concentration of $0.02 \mathrm{mg} / \mathrm{mL}$ in Dulbecco's phosphate-buffered saline (DPBS) at room temperature. $\zeta$-Potential measurements were performed using a nanoparticle concentration of $0.06 \mathrm{mg} / \mathrm{mL}$ in $1 \mathrm{mM} \mathrm{NaCl}$ solution.

Flow Cytometry. Flow cytometry was performed using a Beckman Coulter Gallios cytometer with violet $(405 \mathrm{~nm})$, blue $(488 \mathrm{~nm})$, green $(561 \mathrm{~nm})$, and red $(640 \mathrm{~nm})$ lasers. For flow cytometry analysis, the cells were resuspended in FACS buffer (DPBS containing 2.5\% fetal bovine serum (FBS) and $0.1 \%$ sodium azide) at a concentration of 1 mio cells $/ \mathrm{mL}$. A total of 10000 events were analyzed per experiment. The gating strategy that was applied for the analysis of the cells is shown in Supporting Information Figure S1. The data were analyzed using Flowjo software.

Transmission Electron Microscopy. A $5 \mu \mathrm{L}$ drop of a $4 \mathrm{mg} / \mathrm{mL}$ solution of the poly(ethylene glycol) (PEG)-PLA nanoparticles in DPBS was adsorbed onto a glow-discharged carbon-coated copper grid (400 mesh, Canemco \& Marivac), washed with deionized water, and stained with $5 \mu \mathrm{L}$ of uranyl acetate (2\%). Observations were made using an F20 electron microscope (Fisher Scientific AG) operated at $200 \mathrm{kV}$. Digital images were collected using an Eagle CCD camera (Fisher Scientific AG) at $4098 \times 4098$ pixels, using a defocus range of -2 to $-5 \mu \mathrm{m}$.

Laser Scanning Confocal Microscopy. Confocal microscopy images were acquired as described in ref $^{52} 52$ on a Zeiss LSM700 microscope with a Plan-Apochromat $63 \times / 1.40$ oil objective. $Z$-stacks were taken with a distance of $130 \mathrm{~nm}$ between each focal plane. The resolution of the images is 26.2 pixel per $\mu \mathrm{m}$, and the voxel size is 38.2 $\times 38.2 \times 130 \mathrm{~nm}^{3}$. Images were acquired sequentially (channel 1 and channel 2 together and channel 3 separately) to avoid excitation and emission bleed-through with the following settings for the individual channels. Channel 1 excitation: $405 \mathrm{~nm}$, detection: 405-490 nm, channel 2 excitation: $555 \mathrm{~nm}$, detection: $555-588 \mathrm{~nm}$, and channel 3 excitation: $488 \mathrm{~nm}$, detection: $488-555 \mathrm{~nm}$. The pinhole was adjusted for each channel individually to obtain the same optical slice thickness of $0.4 \mu \mathrm{m}$. For channels 1 and 2, the pinhole was set to obtain the same optical slice thickness as in channel 3 as $0.33 \mathrm{AU}$. The zoom was adjusted to 1.3 .

Confocal Microscopy Image Analysis. Image analysis was performed according to ref $^{52} 52$. Microscopy images were deconvolved using Huygens Remote manager and processed using ImageJ $1.52 \mathrm{p}$ and Imaris. The matlab code is available in ref 53. Nanoparticle localization with respect to the cell membrane and cell body was analyzed with the help of the Imaris spot detection and a distance transform operation. Nanoparticles are detected as spots based on the 


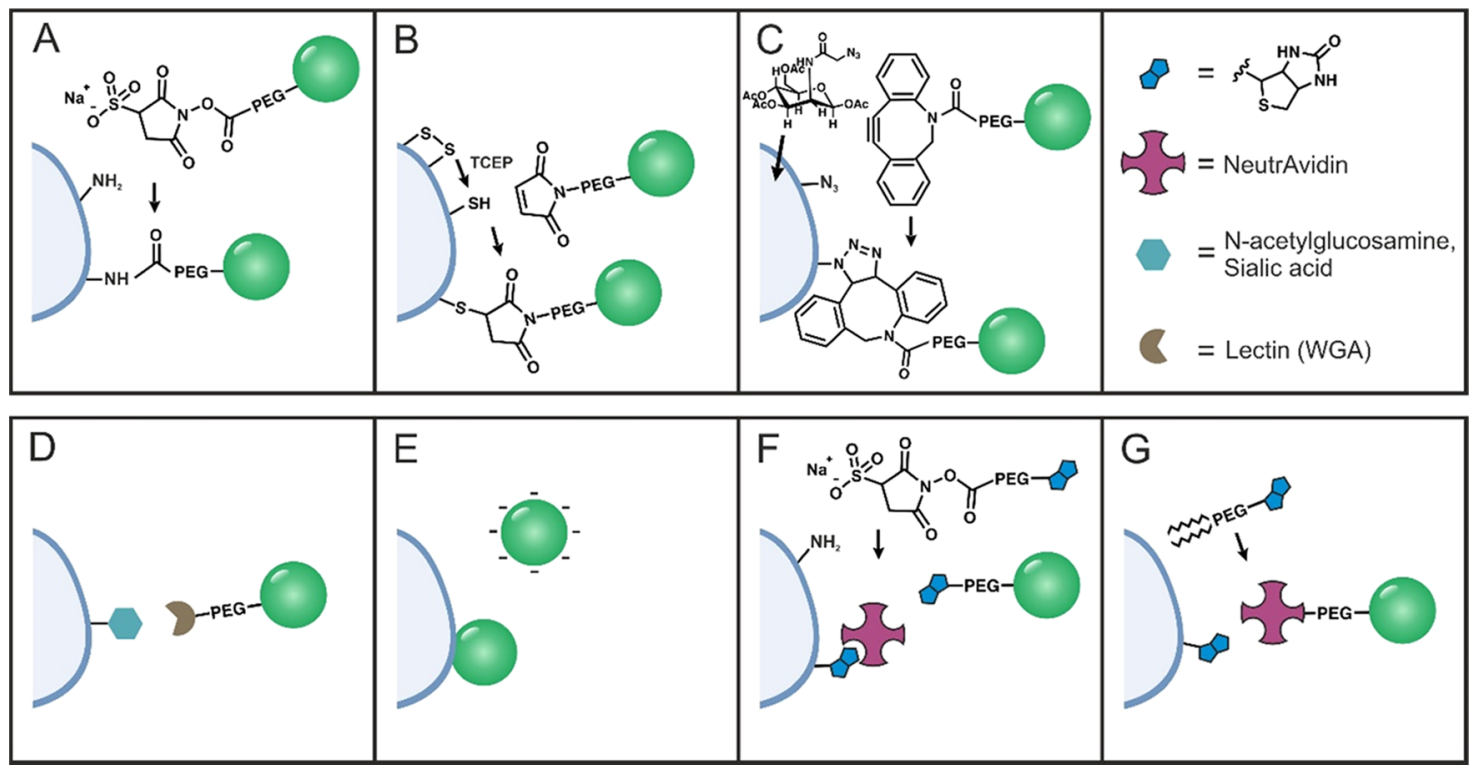

Figure 1. Schematic overview of the nanoparticle cell surface immobilization chemistries explored in this study: (A) Covalent coupling of active ester-modified nanoparticles with surface amino groups; (B) coupling of maleimide-functionalized nanoparticles with thiol groups; (C) click reaction between dibenzocyclooctyne-modified nanoparticles and azido groups on the cell surface; (D) noncovalent binding of WGAfunctionalized nanoparticles to carbohydrates in the glycocalyx; (E) electrostatic adsorption of negatively charged nanoparticles; and biotinNeutrAvidin-mediated immobilization of nanoparticles to cells that are either (F) covalently modified with biotin or (G) modified noncovalently by insertion of a lipid-functionalized biotin moiety before binding of NeutrAvidin nanoparticles to the cell surface.

fluorescence signal above the threshold, and their sizes were measured using an Imaris built-in spot detector (smoothing: 0.15, quality: 20, spot XY: $0.2 \mu \mathrm{m}$, spot Z: $0.4 \mu \mathrm{m}$ (detect ellipsoid), perform region growing, threshold: 2). To quantify the number of nanoparticles per cell, for the image analysis in this paper, an average particle size of 200 $\mathrm{nm}$ was used. Larger spots were considered as aggregates of multiple $200 \mathrm{~nm}$ diameter nanoparticles. Surfaces are detected using an Imaris built-in surface detector (smoothing: 0.25 , surface threshold: 200 (auto), largest sphere: $0.5 \mu \mathrm{m}$, min. volume $80 \mu \mathrm{m}^{3}$ ). Cell bodies are detected using an Imaris built-in surface detector (smoothing: 0.2, surface threshold: 500 (auto), largest sphere $10 \mu \mathrm{m}$, min. volume 100 $\left.\mu \mathrm{m}^{3}\right)$. For each detected surface, a Euclidean distance map is computed. In the distance map, each pixel contains its distance to the nearest surface edge. Then, the average distance to the surface edge for each spot by measuring the mean intensity around the spot in the Euclidean distance map was computed. Negative values represent objects inside the surface, and positive values represent objects outside the surface. Nanoparticle agglomerates were fitted with several nanoparticles based on the nanoparticle size.

Procedures. Cell Surface Immobilization of Nanoparticles. Cells (Jurkat cells and SJL/PLP7 cells) were washed two times with DPBS and then suspended in DPBS at a concentration of 5 mio cells $/ \mathrm{mL}$. Then, the appropriate PEG-PLA or PLA-COOH nanoparticles in DPBS $(1 \mathrm{mg} / \mathrm{mL})$ were added to the cell suspension at the desired nanoparticle/cell ratio and incubated for $30 \mathrm{~min}$ at $37{ }^{\circ} \mathrm{C}$. The reaction mixture was mixed every $10 \mathrm{~min}$ to ensure a homogeneous distribution of the nanoparticles. After immobilization of the nanoparticles on the cell membrane, the cells were washed three times with $10 \mathrm{~mL}$ of DPBS to remove unbound nanoparticles. The mean fluorescent intensities (MFIs) were calculated by subtracting the MFI of the unmodified control cells from that of the nanoparticledecorated cells.

Cell Viability. Viability assays were performed using Annexin VAlexa Fluor 647 and DAPI as a dead cell stain. Briefly, unmodified or surface-modified $\mathrm{T}$ cells (Jurkat cells and SJL/PLP7 cells) were washed once with DPBS and 0.3 mio cells were resuspended in Annexin buffer containing $1 \mu \mathrm{g} / \mathrm{mL}$ DAPI at a concentration of 1 mio cells/mL. Then, $15 \mu \mathrm{L}$ of Annexin V-Alexa Fluor 647 conjugate was added to the cell suspension and cells were incubated at room temperature for $15 \mathrm{~min}$ in the dark. Subsequently, $400 \mu \mathrm{L}$ of Annexin binding buffer was added and cells were directly analyzed by flow cytometry. As a positive control for apoptosis, T cells were incubated overnight in complete growth medium supplemented with $1 \mu \mathrm{M}$ staurosporine. Control cells and staurosporine-treated cells were used to gate cell populations as follows: viable cells were gated as quadrant Q4, early apoptotic cells as quadrant Q1, late apoptotic cells as quadrant Q2, and necrotic cells as quadrant Q3 (Supporting Information Figure S2). Cell viabilities of nanoparticle-decorated cells were determined and normalized by the viability control cell viabilities.

CellTrace Violet Staining. Cells (Jurkat cells and SJL/PLP7 cells) were washed two times with DPBS and resuspended at a concentration of $1 \mathrm{mio} / \mathrm{mL}$ cells. Then, CellTrace violet $(5 \mu \mathrm{M}, 1$ $\mathrm{mg} / \mathrm{mL}$ in dimethyl sulfoxide (DMSO)) was added to the cell suspension and incubated for $20 \mathrm{~min}$. Subsequently, $30 \mathrm{~mL}$ of cell culture medium was added for $5 \mathrm{~min}$ followed by resuspension in cell culture medium and additional incubation for at least $30 \mathrm{~min}$.

Confocal Microscopy Sample Preparation. Samples for confocal microscopy were prepared immediately after the cell surface modification. To a suspension containing 0.5 million CellTrace Violet stained cells (Jurkat cells and SJL/PLP7 cells) at a concentration of 1 mio cells $/ \mathrm{mL}, 25 \mu \mathrm{L}$ of a $1 \mathrm{mg} / \mathrm{mL}$ solution of WGA Texas red in DPBS $(1.4 \mathrm{mM})$ was added. After incubation for 30 min on ice, the cells were washed twice with DPBS and fixed with $4 \%$ paraformaldehyde (PFA) solution in DPBS at room temperature for $20 \mathrm{~min}$. After two washing steps, the cells were resuspended at a concentration of 1 mio cells $/ \mathrm{mL}$ in DPBS and sedimented on a poly(L-lysine)-coated coverslip (diameter $12 \mathrm{~mm}$ ) by centrifugation (200 g, $3 \mathrm{~min}$ ). The supernatant was discarded and the coverslip was mounted with mounting media (ProLong Gold Antifade Mountant) on microscope slides. The slides were cured for $24 \mathrm{~h}$ and sealed.

Flow Cytometry Proliferation Assay. Surface-decorated, CellTrace Violet stained cells were resuspended in cell culture medium at a concentration of 0.5 mio cells $/ \mathrm{mL}$ and incubated for $24 \mathrm{~h}$. The cells were analyzed by FACS analysis both $t=0 \mathrm{~h}$ and after $24 \mathrm{~h}$. Cell proliferation was assessed by comparison of the CellTrace violet mean fluorescence intensity (MFI) at $t=0 \mathrm{~h}$ and after $24 \mathrm{~h}$.

ICAM-1 Binding Assay. The ICAM-1 binding assay of nanoparticle-decorated cells and control cells was performed according to ref 51 . 

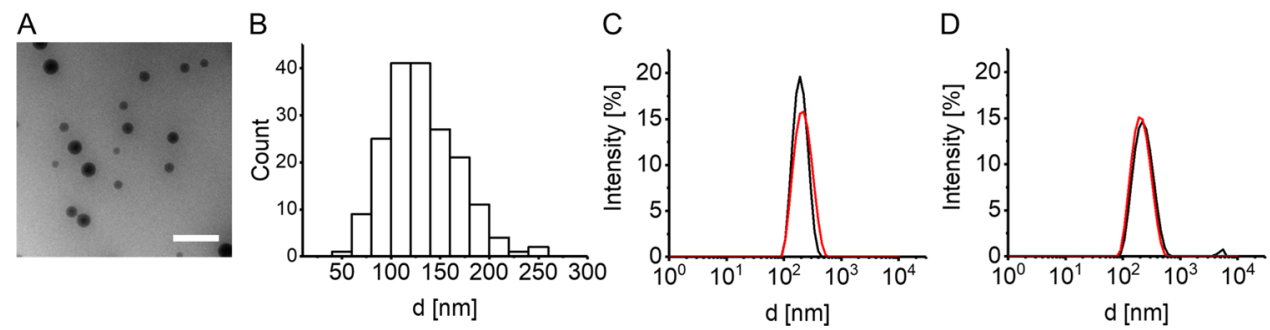

Figure 2. Characterization of amine- and carboxylic acid-functionalized PLA nanoparticles. (A) TEM image of amino-functionalized PEG-PLA nanoparticles (scale bar $=500 \mathrm{~nm})$; (B) intensity-weighted size distribution of the hydrodynamic diameter of amine-functionalized PLA nanoparticles (obtained by TEM analysis of 183 nanoparticles). (C, D) Size distribution of amine-functionalized (C) and carboxylic acidfunctionalized nanoparticles: (black) distribution of plain nanoparticles; (red) distribution of the corresponding DiO-loaded nanoparticles. The data are presented as average of three measurements.

Transendothelial Migration (TEM) Assay. Primary mouse brain microvascular endothelial cells (pMBMECs) from female C57BL/6 mice were seeded on a $6.5 \mathrm{~mm}$ Transwell filter $(5 \mu \mathrm{m}$ pore size $)$ previously coated with laminin and Matrigel. pMBMECs were grown for 7 days to confluency and for 2 days in the presence of puromycin to deplete contaminating pericytes as described. ${ }^{54}$ To prevent the pMBMECs from sprouting through the pores of the filter, they were grown to confluency without medium in the lower compartment. Prior to the experiment, pMBMECs were stimulated with interleukin $1 \beta$ (IL-1 $\beta)(20 \mathrm{ng} / \mathrm{mL})$ for $12 \mathrm{~h}$. At the beginning of the transmigration assay, pMBMEC inserts were washed twice with migration assay medium (MAM: Dulbecco's modified Eagle's medium (DMEM), 2\% L-glutamine, $25 \mathrm{mM} \mathrm{N}$-(2-hydroxyethyl)piperazine- $N^{\prime}$-ethanesulfonic acid (HEPES) 5\% FBS) before being transferred into a new 24-well Costar plate well containing $600 \mu \mathrm{L}$ of MAM. Then, $100 \mu \mathrm{L}$ of MAM containing 100000 nanoparticledecorated SJL-PLP7 T cells were added per insert and T cells were allowed to transmigrate for $6-8 \mathrm{~h}$ at $37^{\circ} \mathrm{C}$. Additionally, aliquots of $100000 \mathrm{~T}$ cells were kept in MAM and used as representative for the input. The number of transmigrated $\mathrm{T}$ cells and the number of $\mathrm{T}$ cells in the input samples were assessed by flow cytometry. The percentage of migrated $\mathrm{T}$ cells was calculated referring to the input as $100 \%$. Finally, the inserts were washed twice in PBS and fixed in 37\% PFA vapor for $2 \mathrm{~h}$. Fixed inserts were blocked for $20 \mathrm{~min}$ in a blocking buffer and stained for $1 \mathrm{~h}$ at room temperature with Phalloidin Rhodamine (stock solution 300 units in $1.5 \mathrm{~mL}$ of methanol, 1: 200) and CD45 biotin $(1: 50,10 \mu \mathrm{g} / \mathrm{mL})$. After three washing steps with PBS, Streptavidin-Cy $5(1: 100,15 \mu \mathrm{g} / \mathrm{mL})$ was added for $1 \mathrm{~h}$ at room temperature. Then, the filters were washed again with PBS and the cell nuclei were stained with DAPI in blocking buffer (1:2000, $0.5 \mathrm{ng} /$ $\mathrm{mL}$ ). The inserts were mounted with Mowiol on glass slides, and the confluency of the endothelial monolayer was confirmed with immunofluorescence microscopy imaging of each filter after each assay.

\section{RESULTS AND DISCUSSION}

Nanoparticle Preparation. As a model system to investigate the impact of various surface conjugation chemistries on the viability and functional properties of cellbased carriers, this study uses blood-brain barrier migratory $\mathrm{T}$ cells that will be modified with poly(D,L-lactic acid) (PLA) nanoparticles displaying a range of cell surface reactive chemistries. As they are nonphagocytic, the risk of nanoparticle internalization by these $\mathrm{T}$ cells is minimal, which makes them attractive models to study cell surface functionalization. For this study, two $\mathrm{T}$ cell lines were investigated. Jurkat cells, which are an immortalized class of $\mathrm{T}$ cells, were used as one model. The second class of $\mathrm{T}$ lymphocytes studied were SJL/PLP7 cells. This is a proteolipid protein (PLP)-specific cell line, which is used to study inflammatory $\mathrm{T}$ cell infiltration of the central nervous system (CNS). ${ }^{55,56}$ In this paper, mice-derived
SJL/PLP7 CD4 ${ }^{+} \mathrm{T}_{\mathrm{EM}}$ cells were explored as model carriers to transport polymer nanoparticles across the blood-brain barrier. Figure 1 presents the different surface-functionalized nanoparticles and cell surface conjugation chemistries that will be explored. The nanoparticles will be immobilized using both covalent and noncovalent strategies. Covalent cell surface conjugation chemistries that will be assessed include the use of active ester-functionalized PLA nanoparticles that can react with cell surface amino groups (Figure 1A), the coupling of maleimide-functionalized PLA nanoparticles with thiol groups present on the cell surface (Figure 1B) as well as the bioorthogonal, strain-promoted azide-alkyne cycloaddition of alkyne-functionalized nanoparticles to cell surface azido groups (Figure 1C). Noncovalent cell surface conjugation chemistries that will be examined are the binding of Wheat germ agglutinin (WGA)-modified PLA nanoparticles to $\mathrm{N}$-acetylglucosamine and sialic acid residues on the cell surface (Figure 1D), the electrostatic binding of negatively charged PLA nanoparticles (Figure 1E) as well as the biotin-NeutrAvidin-mediated immobilization of PLA nanoparticles. The latter can be achieved either by using biotin moieties that are covalently coupled to cell surface amino groups (Figure $1 \mathrm{~F}$ ) or by noncovalent insertion of biotin-functionalized PEGylated lipids (Figure 1G). The binding of negatively charged nanoparticles to the cell surface (Figure 1E) can be due to one or a combination of two mechanisms. A first one is based on electrostatic interactions between the negatively charged nanoparticles and cationic charges on the cell membrane. ${ }^{57-59}$ A second mechanism that can promote binding of negatively charged nanoparticles onto the cell membrane is entropy gaindriven depletion. ${ }^{6-62}$

Carboxylic acid- and amine-functionalized PLA nanoparticles were prepared via nanoprecipitation. Carboxylic acid-functionalized PLA nanoparticles were obtained by precipitation of an acetone solution containing carboxylic acid terminated poly(D,L-lactic acid) in DPBS. Aminofunctionalized PLA nanoparticles were obtained by coprecipitation of equal amounts of methoxy-terminated poly(D,L-lactic acid) and an amino-terminated poly(D,L-lactic acid)$b$-poly (ethylene glycol) block copolymer (PLA-PEG- $\mathrm{NH}_{2}$ ). If needed, nanoparticles were loaded with the green fluorescent dye $\mathrm{DiO}$ to allow analysis by flow cytometry and confocal microscopy. The nanoparticles were characterized by dynamic light scattering (DLS) and transmission electron microscopy (TEM).

Figure $2 \mathrm{~A}-\mathrm{C}$ presents the results for the amine-functionalized PLA nanoparticles. TEM analysis reveals spherical particles with a number-average diameter of $130 \pm 36 \mathrm{~nm}$ 
Scheme 1. Surface Modification of Amine-Functionalized PLA Nanoparticles
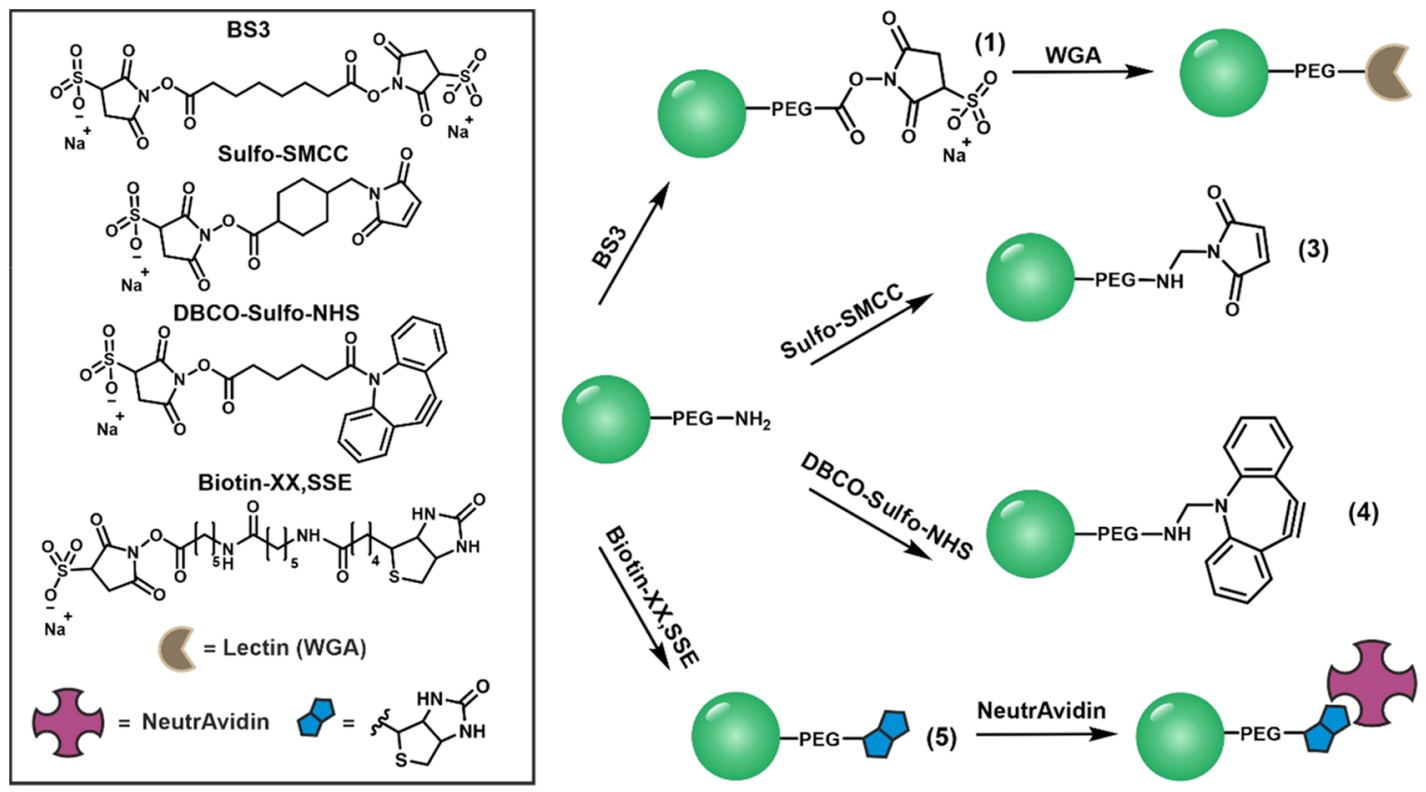

(based on the analysis of 183 nanoparticles in total) (Figure 2A,B). This is in reasonable agreement with the hydrodynamic diameter of $185.7 \pm 3.5 \mathrm{~nm}$ and the PDI of 0.1 obtained from DLS analysis. These nanoparticles had a near-neutral $\zeta$ potential (Supporting Information Figure S3). The aminefunctionalized PLA nanoparticles could be loaded with $150 \mathrm{ng}$ $\mathrm{DiO} / \mathrm{mg}$ polymer without significantly affecting the particle size (Figure 2C) but with a slight increase in PDI from 0.6 to 0.8 (Supporting Information Figure S3). ${ }^{1} \mathrm{H}$ NMR analysis revealed that these nanoparticles were composed of $7.5 \mathrm{~mol} \%$ PLA-PEG-NH $\mathrm{N}_{2}$ (Supporting Information Figure S4A). By reacting the nanoparticles with NHS fluorescein, the surface concentration of amino groups was estimated at $2.9 \mathrm{nmol} / \mathrm{mg}$. Results of the DLS analyses of the carboxylic acid-functionalized PLA nanoparticles are presented in Figure 2D. These particles are characterized by a hydrodynamic diameter of $205.3 \pm 1.3 \mathrm{~nm}$, a PDI of 0.17 , and a $\zeta$-potential of $-30.4 \mathrm{mV}$ (Supporting Information Figure S3). The carboxylic acidfunctionalized nanoparticles could be loaded with $475 \mathrm{ng}$ $\mathrm{DiO} / \mathrm{mg}$ polymer, corresponding to an encapsulation efficiency of $95 \%$, without altering the nanoparticle size or size distribution (Figure 2D).

To introduce cell surface reactive $N$-hydroxysulfosuccinimide (NHS) (Figure 1A), maleimide (Figure 1B), dibenzocyclooctyne (DBCO) (Figure 1C), or biotin moieties (Figure $1 \mathrm{~F}$ ), or to attach WGA (Figure 1D) or NeutrAvidin (Figure $1 \mathrm{G})$, the amine-functionalized nanoparticles were modified as shown in Scheme 1. These surface modification reactions were performed by treatment of the amine-functionalized nanoparticles with the appropriate sulfo-NHS reagent $(\mathbf{1}, \mathbf{3}, \mathbf{4}, \mathbf{5})$. WGA (2)- and NeutrAvidin-functionalized nanoparticles (6) were obtained by reaction of the sulfo-NHS (1), respectively, biotin-functionalized (5), precursor particles with the respective protein. The surface-modified nanoparticles were analyzed by DLS and $\zeta$-potential measurements (Supporting Information Figure S3). These analyses indicated that the various surface modification reactions did not significantly influence the size, PDI, or $\zeta$-potential of the nanoparticles compared to the amine-functionalized precursor particles. Only for the DBCO and NeutrAvidin-modified nanoparticles, a slight increase in hydrodynamic diameter and PDI was observed, which could be due to an increased surface hydrophobicity of the nanoparticles. To determine the concentration of cell surface reactive groups, the maleimidefunctionalized nanoparticles were modified with a thiolcontaining Cy5 derivative. Spectrophotometric analysis of the resulting nanoparticles revealed a maleimide concentration of $0.4 \mathrm{nmol} / \mathrm{mg}$ polymer (Supporting Information Figure S5C). The nanoparticle surface concentrations of biotin, NeutrAvidin, and WGA were obtained similarly using Cy5-streptavidin, Cy5 biotin, and Cy5-WGA and determined as 0.6, 0.2, and $0.02 \mathrm{nmol} / \mathrm{mg}$, respectively (Supporting Information Figure S5C).

A number of nanoparticle immobilization strategies that are summarized in Figure 1 require preactivation or premodification of the cell surface. To increase the number of free surface thiol groups, cells that were reacted with maleimide-functionalized nanoparticles were preactivated with tris(2carboxyethyl)phosphine (TCEP). To establish reaction conditions to maximize the concentration of surface thiol groups, Jurkat cells were treated with a range of TCEP concentrations up to $10 \mathrm{mM}$ and the concentration of surfaceaccessible thiol groups was determined using Ellman's reagent. Activation with TCEP increases the surface concentration of thiol groups from $\sim 18 \mathrm{nmol} / 10^{6}$ cells for untreated cells to $\sim 36 \mathrm{nmol} / 10^{6}$ cells when cells are treated with $10 \mathrm{mM}$ TCEP (Supporting Information Figure S6). As higher TCEP concentrations have been reported to impact cell viability, ${ }^{63}$ a $10 \mathrm{mM}$ TCEP solution was used to pretreat cells for thiolmaleimide conjugation of PLA nanoparticles. The strainpromoted azide-alkyne cycloaddition of dibenzocyclooctynefunctionalized nanoparticles to the cell surface requires cell surface-bound azide groups, which were introduced via metabolic glycoengineering. Supporting Information Figure S7 presents the results from confocal microscopy and flow cytometry analysis of azide-modified cells and unmodified control cells after treatment with a DBCO-modified Cy5-dye, which confirm the chemoselective, bio-orthogonal click modification of the azide group presenting Jurkat cells. Finally, biotin moieties were introduced to the cell surface either via 

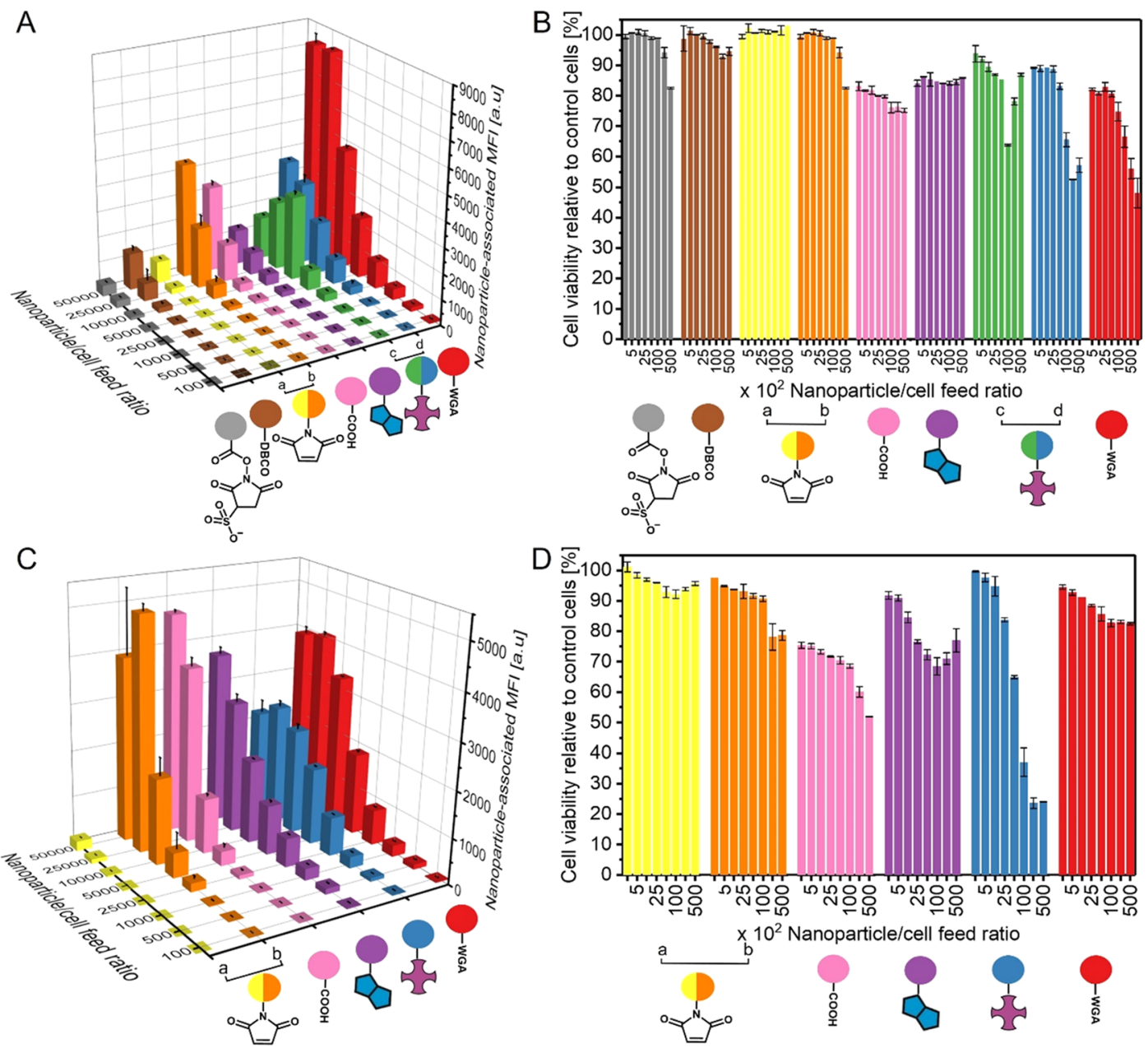

Figure 3. Nanoparticle-associated fluorescence (A, C) and viabilities (B, D) of nanoparticle-decorated Jurkat cells (A, B) and SJL/PLP7 cells (C, D) obtained by flow cytometry. Cells were incubated at initial nanoparticle/cell feed ratios of 100, 500, 1000, 2500, 5000, 10 000, 25 000, and 50 000. Sulfo-NHS-functionalized PLA nanoparticles are shown in gray, DBCO-modified PLA nanoparticles in brown, maleimide-functionalized PLA nanoparticles in yellow/orange $(\mathrm{a}=$ untreated cells; $\mathrm{b}=$ TCEP-treated cells), carboxylic acid-modified PLA nanoparticles in pink, biotinfunctionalized PLA nanoparticles in violet, NeutrAvidin-modified PLA nanoparticles in green/blue $(c=$ noncovalent biotin- and $d=$ covalent biotin cell surface modification) and WGA-functionalized PLA nanoparticles are shown in red. Data are expressed as mean \pm SD $(n=2)$.

covalent coupling of Biotin-XX, SSE or by noncovalent insertion of DSPE-PEG2000-biotin. To validate the successful covalent surface attachment of biotin, the resulting cells were treated with NeutrAvidin-Oregon Green 488 conjugate and analyzed with flow cytometry and confocal microscopy. As a control experiment, also Jurkat cells that were not reacted with Biotin-XX, SSE or DSPE-PEG2000-biotin were treated with NeutrAvidin-Oregon Green 488 conjugate and analyzed in the same way. The results of these analyses, which are presented in Supporting Information Figure S8, confirm the successful coupling of Biotin-XX, SSE. Cells, which were not treated with Biotin-XX, SSE did not show any fluorescent signal in FACS or confocal microscopy confirming the specific interaction between the NeutrAvidin-modified dye and biotinmodified cells. Supporting Information Figure S9 presents the results of similar experiments that were performed to characterize cells that were modified with DSPE-PEG2000biotin and which validate the noncovalent biotinylation of the Jurkat cells. To semiquantitatively assess and compare the relative amounts of the various reactive groups on the cell surface, the modified cells were labeled with the corresponding reactive Cy5 dyes (sulfo-Cy5 NHS ester, sulfo-Cyanine 5 maleimide, Streptavidin Cy5, Cy5 biotin, sulfo-Cyanine 5 DBCO, WGA Cy5) and analyzed by flow cytometry. Supporting Information Figure S10 presents these relative cell surface concentrations, which are presented as the shift in the mean $\mathrm{Cy5}$-associated fluorescent intensity of the modified cells relative to that of the nonmodified, control cells. These analyses reveal that Jurkat cells modified by metabolic glycoengineering present azido groups at concentrations that are comparable to those of the surface amine groups and WGA reactive saccharide residues. It is worth mentioning, however, that the procedure used for the introduction of the cell surface azido groups was not optimized and can probably be further refined to enhance the surface concentration of these bioorthogonal anchors. Jurkat cells treated with TCEP, or covalently or noncovalently modified with biotin also present comparable concentrations of surface-accessible, nanoparticle reactive groups, albeit at a lower concentration compared to amine, azide, and WGA reactive groups. Unmodified Jurkat cells only present a relatively low concentration of thiol groups and immobilization of NeutrAvidin also results in the introduction of fewer nanoparticle reactive groups compared to most other cell preactivation or premodification approaches. 
Cell Surface Modification. In a first series of experiments, the ability of the various covalent and noncovalent conjugation chemistries illustrated in Figure 1 for the cell surface immobilization of polymer nanoparticles was compared using flow cytometry. To explore whether and to which extent the nanoparticle cell surface concentration can be tuned, the cells were incubated with the appropriate nanoparticle at nanoparticle/cell ratios that ranged from 100 to 50000 , then treated with DAPI and Annexin V-Alexa Fluor 647 conjugate, and subsequently analyzed by flow cytometry. In these experiments, the nanoparticle-associated fluorescence observed in flow cytometry was taken as a measure of the nanoparticle cell surface concentration. By staining the cells with DAPI and Annexin V-Alexa Fluor 647 conjugate directly after nanoparticle conjugation, flow cytometry analysis allows to simultaneously assess the viability of the nanoparticledecorated cells. These experiments were performed with both Jurkat cells and SJL/PLP7 cells.

Figure $3 \mathrm{~A}, \mathrm{~B}$ summarizes the results that were obtained for the surface modification of Jurkat cells. At a given nanoparticle/cell ratio, most noncovalent nanoparticle coupling strategies result in more pronounced shifts in nanoparticleassociated fluorescence, i.e., higher nanoparticle cell surface concentrations, compared to the covalent chemistries that were investigated (Figure 3A). From the covalent chemistries that were screened, coupling of NHS and DBCO-functionalized nanoparticles resulted in the lowest nanoparticle cell surface concentrations. Conjugation of maleimide-functionalized nanoparticles could be significantly enhanced by pretreating the Jurkat cells with TCEP, which reduces surface disulfides and increases the concentration of surface thiol groups. In most cases, increasing the initial nanoparticle/cell ratio leads to an increase in the nanoparticle-associated fluorescence, which indicates that the nanoparticle cell surface concentration can be tuned by adjusting the nanoparticle/cell ratio. Judging from the observed shifts in the nanoparticle-associated fluorescence that were measured by flow cytometry, WGA-mediated nanoparticle cell surface immobilization is most effective, i.e., results in the highest cell surface nanoparticle concentrations. The flow cytometry analysis also suggests that the use of NeutrAvidin-functionalized nanoparticles provides an efficient, alternative strategy for the surface modification of the Jurkat cells. For cells that were modified by covalent attachment of biotin, a continuous increase in nanoparticle-associated mean fluorescence intensity (MFI) with increasing initial nanoparticle/cell ratio was observed. For Jurkat cells that presented biotin, which was introduced by modifying the cells with DSPE-PEG2000-biotin, in contrast, the nanoparticle-associated fluorescence was found to increase upon increasing the initial nanoparticle/cell ratio from 100 to 1000 but then decrease at initial nanoparticle/cell ratios of 25000 and 50000 . Presenting cells with the amine-functionalized PEGylated PLA nanoparticles resulted in essentially negligible attachment of nanoparticles to the cell surface (in contrast to that observed when carboxylic acid-functionalized PLA nanoparticles were used, see Figure 3A,C). Most nanoparticle conjugation chemistries did not significantly affect the viability of the Jurkat cells, not even for initial nanoparticle/cell ratios of up to 50000 (Figure 3B). Exceptions are the conjugation of NeutrAvidin-functionalized or WGA-functionalized nanoparticles, where cell viabilities were found to decrease to $\sim 50 \%$ at very high initial nanoparticle/cell ratios.
Figure 3C,D summarizes the results that were obtained with the SJL/PLP7 cells. Since the coupling of active ester and DBCO-functionalized nanoparticles to Jurkat cells did not prove to be effective, these approaches were not assessed on the SJL/PLP7 cells. The results of the surface modification experiments with SJL/PLP 7 cells resemble those obtained with the Jurkat cells and indicate that the nanoparticle surface concentration can be controlled by varying the initial nanoparticle/cell ratio. As it was also observed for the Jurkat cells, pretreatment of the SJL/PLP7 cells with TCEP is important to enhance coupling of maleimide-functionalized nanoparticles. The DAPI/Annexin V viability assay results indicate that the SJL/PLP7 cells are more sensitive toward the cell surface conjugation of maleimide-, carboxylic acid-, and NeutrAvidin-functionalized nanoparticles compared to the Jurkat cells. The viabilities of SJL/PLP7 cells modified with biotin-functionalized nanoparticles were only slightly lower than those of their Jurkat counterparts. For SJL/PLP7 cells modified with WGA-functionalized nanoparticles, viabilities were higher compared to those of the corresponding Jurkat cells.

From the nanoparticle immobilization chemistries that were screened in the experiments discussed above, four were selected and investigated in more detail. The second series of experiments were carried out with maleimide-, biotin-, WGA-, and carboxylic acid-functionalized nanoparticles. The aim of these experiments was to quantitatively study the concentration and localization of the nanoparticles on the cell surface and to compare the proliferation and functional properties of $\mathrm{T}$ cells decorated with nanoparticles using these different chemistries.

Confocal Microscopy. The concentration and localization of the nanoparticles on the cell surface were investigated with confocal microscopy. For these experiments, Jurkat cells were modified with DiO-labeled nanoparticles at initial nanoparticle/cell ratios of 500, 2500, and 5000. To allow confocal microscopy analysis, before surface modification with the PLA nanoparticles, the cells were stained with CellTrace Violet to visualize the cytosol and with WGA Texas Red to highlight the cell membrane. Nanoparticle-decorated cells were analyzed by three-dimensional (3D) reconstructing $z$-stacks recorded from single cells by confocal microscopy. The number of nanoparticles per cell and the location of the nanoparticles on the cell surface were determined via an Imaris script, which allows automated spot detection and distance mapping. ${ }^{52,53}$ The results of these analyses are presented in Figures 4 and 5 . Figure 4 shows representative confocal images of Jurkat cells modified with the four different nanoparticles at nanoparticle/ cell ratios of 500, 2500, and 5000. In these images, the nanoparticles can be clearly identified as green fluorescent spots. For each of the nanoparticle immobilization chemistries, the nanoparticle cell surface coverage was found to increase with increasing initial nanoparticle/cell ratio. The highest nanoparticle cell surface concentrations were observed for the biotin- and WGA-functionalized nanoparticles. At an initial nanoparticle/cell ratio of 5000, cell surface modification with biotin-functionalized nanoparticles resulted in a surface coverage of $184 \pm 56$ nanoparticles/cell, while the use of WGA-functionalized nanoparticles generated cells that carry $176 \pm 44$ nanoparticles/cell. Without preactivation of the surface thiol groups with TCEP, modification of Jurkat cells with maleimide-functionalized nanoparticles results in a nanoparticle surface concentration of $76 \pm 37$ nanoparticles/ 


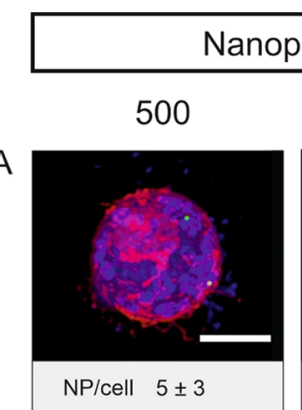

B

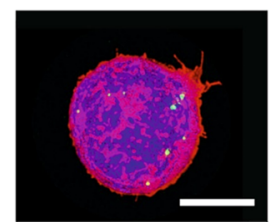

NP/cell $12 \pm 7$

C

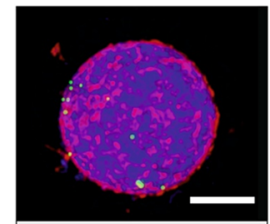

NP/cell $13 \pm 13$
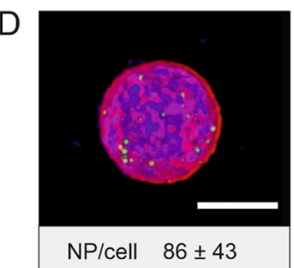

E

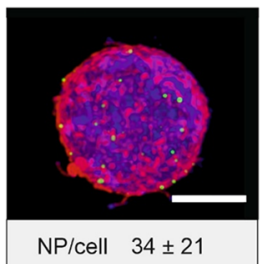

2500

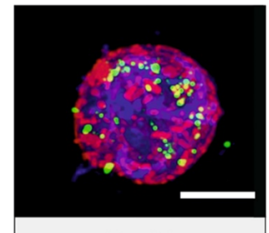

$36 \pm 24$

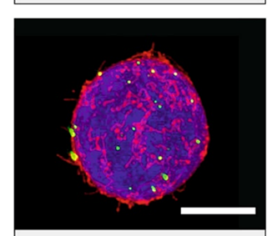

$57 \pm 64$

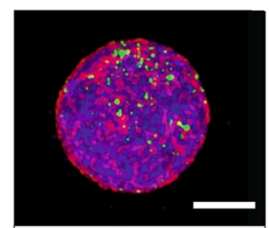

$80 \pm 58$

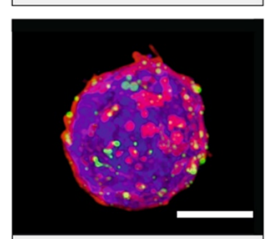

$174 \pm 57$

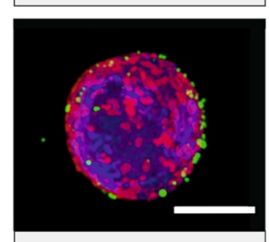

$89 \pm 34$
5000

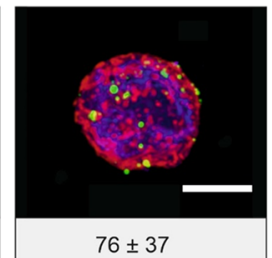

$76 \pm 37$

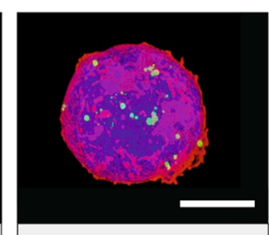

$104 \pm 65$

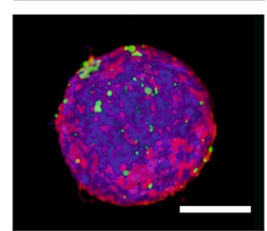

$106 \pm 51$

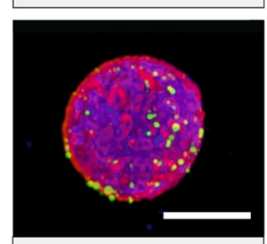

$184 \pm 56$

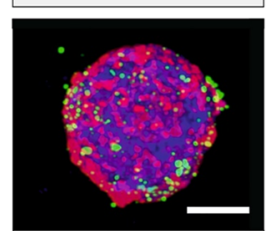

$176 \pm 44$

Figure 4. Confocal laser scanning microscopy images of Jurkat cells, which were incubated with nanoparticles at nanoparticle/cell ratios of 500, 2500, and 5000: (A) non-TCEP-pretreated, maleimide-functionalized PLA nanoparticle-modified cells; (B) TCEP-pretreated and maleimide-functionalized PLA nanoparticle-modified cells; (C) carboxylic acid-functionalized PLA nanoparticle-modified cells; (D) biotin-functionalized PLA nanoparticle-modified cells; and (E) WGAfunctionalized PLA nanoparticle-modified cells. The cell cytosol shown in blue was stained with CellTrace violet, and the membrane, which is shown in red, was stained with WGA Texas Red. Nanoparticles were loaded with the green dye DiO. The average number of nanoparticles per condition was calculated over at least 10 different cells. The scale bars represent $5 \mu \mathrm{m}$.

cell. Pretreating the cells with TCEP results in an increase in the surface concentration of maleimide-functionalized PLA nanoparticles to $104 \pm 65$. This surface concentration is comparable to that obtained when Jurkat cells are modified with carboxylic acid-functionalized nanoparticles at a nanoparticle/cell ratio of $5000(106 \pm 51)$. To put these numbers into perspective, the nanoparticle cell surface concentrations as determined by confocal microscopy correspond to an

estimated $1 \%$ coverage of the total available surface area of a cell.

In addition to the quantitative determination of the surface concentration of the nanoparticles, confocal microscopy was also used to study the localization of the nanoparticles. To this end, images were analyzed with Imaris to create distance maps, which illustrate the distance between a nanoparticle and the cell membrane. The results of these analyses for each of the different nanoparticle cell surface conjugation chemistries investigated are summarized in Figure 5A-E. Figure 5F shows the percentage of WGA Texas Red-positive nanoparticles, i.e., the percentage of nanoparticles that co-localize with the cell membrane for the different cell surface immobilization chemistries. For most of the investigated reaction conditions, the majority of nanoparticles was found to co-localize with the cell membrane. Surface modification of non-TCEP-pretreated Jurkat cells with maleimide-functionalized nanoparticles at nanoparticle/cell ratios of 2500 and 5000 resulted in a significant increase in the fraction of nanoparticles that were attached to the periphery of the cell compared to cells that were modified with the same nanoparticles at an initial nanoparticle/cell ratio of 500 . Exposing TCEP-pretreated Jurkat cells with maleimidefunctionalized nanoparticles at initial nanoparticle/cell ratios of 2500 and 5000, in contrast, resulted in significant internalization of the maleimide-functionalized nanoparticles compared to surface modification reactions that were performed at an initial nanoparticle/cell ratio of 500. For TCEP-treated Jurkat cells that were modified with maleimidefunctionalized nanoparticles at a nanoparticle/cell ratio of 500, $90 \%$ of the nanoparticles were found to co-localize with the cell membrane. For reactions that were carried out with nanoparticle/cell ratios of 2500 and 5000, only 71 and $60 \%$ of the nanoparticle payload were found to co-localize with the cell membrane, respectively. Surface modification of Jurkat cells with carboxylic acid-functionalized nanoparticles, in particular at higher nanoparticle/cell ratios, also resulted in the immobilization of a significant fraction of nanoparticles at the periphery of the cells as well as nanoparticle internalization. In contrast, when Jurkat cells were modified with biotin- or WGA-functionalized nanoparticles, $\sim 90 \%$ of the nanoparticles were found to be co-localized with the cell membrane with very little to no internalization and only a very small fraction bound to the periphery of the cell membrane, irrespective of the initial nanoparticle/cell ratio.

Confocal microscopy was also used to study SJL/PLP7 cells that were modified with biotin-functionalized nanoparticles at an initial nanoparticle/cell ratio of 5000 (Figure 6A,B). SJL/ PLP7 cells modified using these conditions carry $153 \pm 52$ nanoparticles/cell, which compares well with the nanoparticle cell surface concentrations that were found for Jurkat cells modified under the same conditions (Figure 4). Distance mapping of the nanoparticle-decorated SJL/PLP7 cells, however, reveals that only $48 \%$ of the nanoparticles co-localize with the cell membrane and also reveals nanoparticle internalization as opposed to what was observed for the corresponding Jurkat cells (Figure 5D).

Proliferation. In a next series of experiments, the proliferation of Jurkat $\mathrm{T}$ cells decorated with maleimide-, carboxylic acid-, biotin-, and WGA-functionalized nanoparticles was compared with that of unmodified Jurkat cells. These experiments were carried out with cells modified with nanoparticles at initial nanoparticle/cell ratios of 500, 2500, 
A

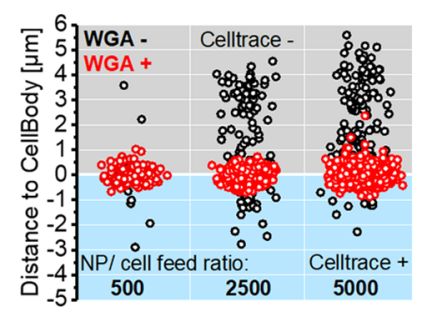

D

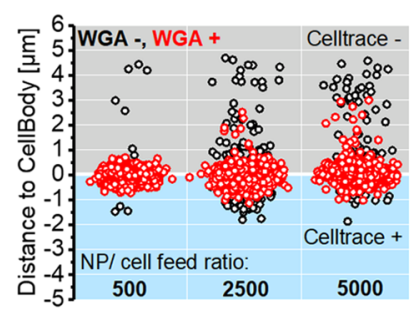

B

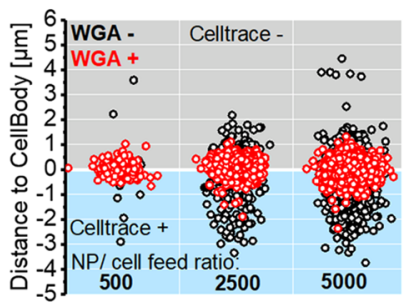

$\mathrm{E}$

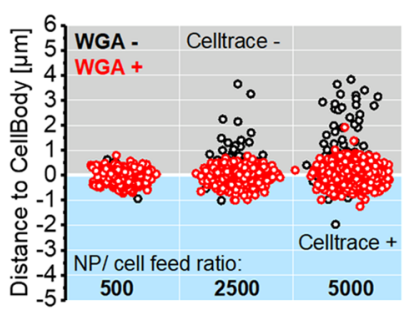

C

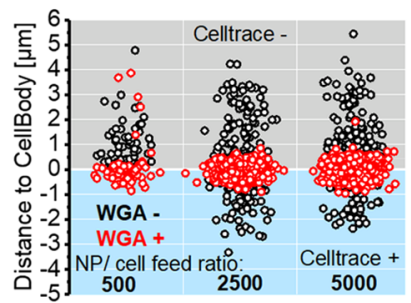

$\mathrm{F}$

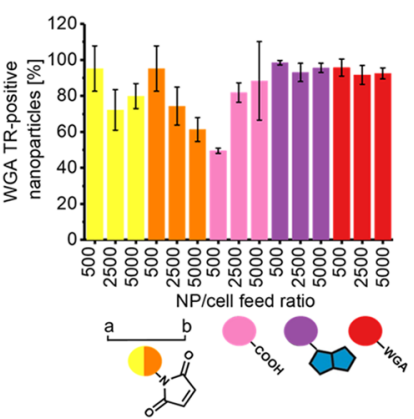

Figure 5. Distance analysis of nanoparticle distribution on Jurkat cells. (A-E) Statistical distribution of nanoparticles on at least 10 cells with respect to their distance to the whole cell edge (CellTrace Violet). Data shown are for cells modified at nanoparticle/cell ratios of 500, 2500, and 5000. Each dot represents a single nanoparticle. Red dots represent nanoparticles that are found between the inner and outer boundaries outlined by the membrane stain (WGA Texas Red X), and black dots represent those which are not. (A) Non-TCEP-pretreated cells decorated with maleimide-functionalized PLA nanoparticles; (B) TCEP-pretreated cells decorated with maleimide-functionalized PLA nanoparticles; (C) cells modified with carboxylic acid-functionalized PLA nanoparticles; (D) cells modified with biotin-functionalized PLA nanoparticles; (E) cells modified with WGA-functionalized PLA nanoparticles; and (F) percentage of nanoparticles that co-localize with the WGA Texas Red membrane stain at initial nanoparticle/cell ratios of 500, 2500, and 5000. Yellow: maleimide nanoparticle-modified cells, orange: maleimide nanoparticlemodified and TCEP-treated cells, rose: carboxylic acid-functionalized nanoparticle-modified cells; and red: WGA-nanoparticle-modified cells.

A

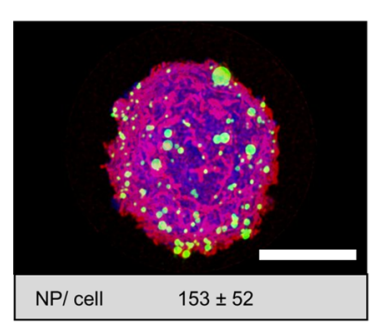

B

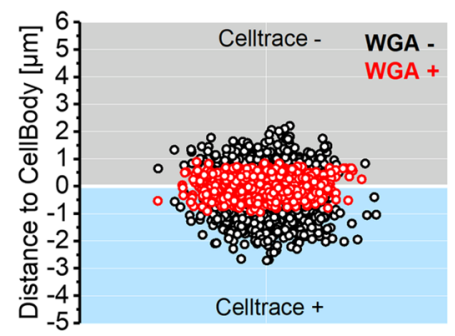

Figure 6. Analysis of SJL/PLP7 cells, which were modified with NeutrAvidin and decorated with biotin-functionalized PLA nanoparticles at an initial feed ratio of 5000 nanoparticles/cell. (A) Representative confocal microscopy image. The cell cytosol shown in blue was stained with CellTrace violet, and the membrane, which is shown in red, was stained with WGA Texas Red, the green nanoparticles are loaded with $\mathrm{DiO}$ (scale bar $=5 \mu \mathrm{m}$ ). (B) Nanoparticle localization relative to the cell body and the cell membrane. Nanoparticles that co-localize with the cell membrane are shown as red dots (WGA+), and nanoparticles that do not co-localize with the cell membrane stain are shown as black spheres (WGA-).

and 5000. Proliferation was analyzed by flow cytometry by staining cells with CellTrace Violet and comparing the CellTrace Violet-associated fluorescence directly after cell surface modification and after $24 \mathrm{~h}$. Cell proliferation was expressed as the ratio of the mean CellTrace Violet-associated fluorescence intensity (CTV-MFI) at $t=0$ divided by the mean CellTrace Violet-associated fluorescence intensity at $t=$ $24 \mathrm{~h}$. Figure 7A presents the ratios of these CellTrace Violet MFIs at $t=0$ and $24 \mathrm{~h}$ for unmodified control cells together with those of the nanoparticle-decorated cells. For the nonmodified control cells, the CellTrace Violet MFI at $t=0$ is twice that at $t=24 \mathrm{~h}$, which is consistent with a single proliferation cycle. Modification of Jurkat cells with maleimidefunctionalized nanoparticles (without prior TCEP pretreatment) or with carboxylic acid- or WGA-functionalized nanoparticles at initial nanoparticle/cell ratios of 500, 2500, and 5000 does not significantly change cell proliferation as compared to the unmodified control cells. For Jurkat cells pretreated with TCEP before conjugation of maleimidefunctionalized nanoparticles, a slight increase in proliferation was observed, while for cells modified with biotin-functionalized nanoparticles, proliferation appeared to be slightly decreased compared to the control cells.

Figure $7 \mathrm{~B}$ compares the proliferation, expressed as the ratio of the mean CellTrace Violet-associated fluorescence intensity at $t=0$ divided by the mean CellTrace Violet-associated fluorescence intensity at $t=24 \mathrm{~h}$, of SJL/PLP7 cells modified with carboxylic acid-, biotin-, and WGA-functionalized nanoparticles at an initial nanoparticle/cell ratio of 5000 with that of nonmodified control cells. The results summarized in Figure $7 \mathrm{~B}$ indicate that cell surface modification under these conditions does not affect the proliferation of SJL/PLP7 cells.

ICAM-1 Binding and Migration Across an In Vitro BBB Model. To study the influence of nanoparticle cell surface modification on the migratory properties of cells, SJL/PLP7 cells were used. This subset of $\mathrm{T}$ cells was chosen as it possesses the ability to cross the $\mathrm{BBB}$, which makes them of potential interest for the cell-mediated delivery of nanomedicines to the CNS. ${ }^{51}$ The $\mathrm{BBB}$ migratory properties of the SJL/PLP7 cells were evaluated in two experiments. In a first experiment, the binding of unmodified SJL/PLP7 cells and 

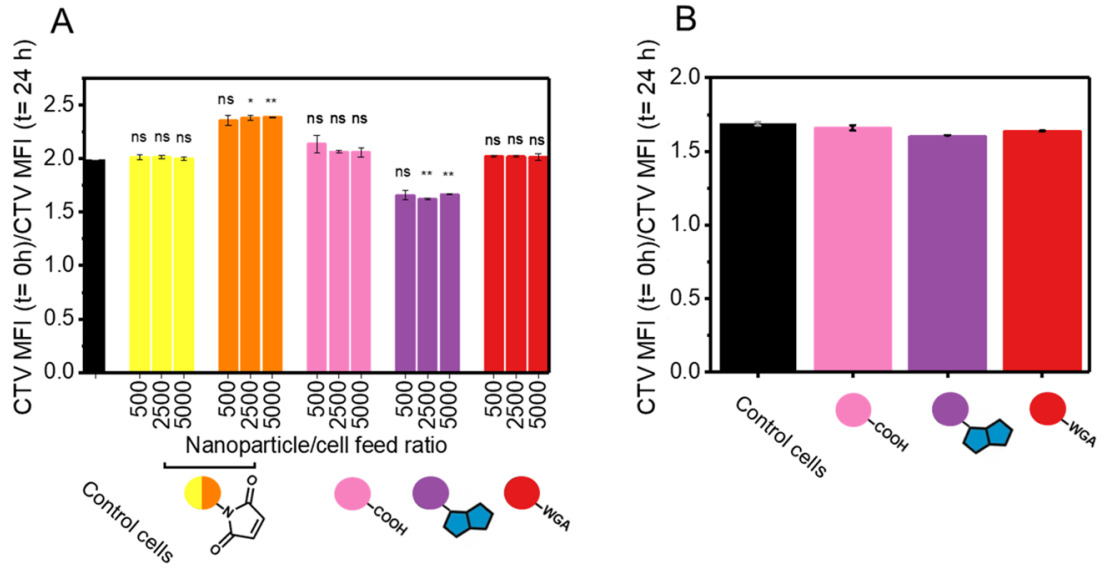

Figure 7. (A) Proliferation of Jurkat T cells, presented as mean fluorescence intensity (MFI) decrease of CellTrace Violet (CTV) over a period of $24 \mathrm{~h}$. Control, unmodified cells are illustrated in black, maleimide-functionalized PLA nanoparticle-decorated cells in yellow, maleimidefunctionalized nanoparticle-decorated cells after TCEP pretreatment in orange, carboxylic acid-functionalized PLA nanoparticle-decorated cells in pink, biotin-functionalized PLA nanoparticle-decorated cells in violet, and WGA-functionalized PLA nanoparticle-decorated cells in red. Cells were decorated with nanoparticles using initial nanoparticle/cell ratios of 500, 2500, and 5000 nanoparticles/cell. P-values were determined by a twotailed $t$-test: $\mathrm{ns}=$ not significant, $*=P \leq 0.05$ and $* *=P \leq 0.01$. Data are expressed as mean $\pm \mathrm{SD}(n=2)$. (B) Cell proliferation during $24 \mathrm{~h}$ of control SJL/PLP7 cells (black), carboxylic acid-functionalized PLA nanoparticle-decorated cells (rose), biotin-functionalized nanoparticledecorated cells (violet), and WGA-functionalized nanoparticle-modified cells (red) analyzed by a CellTrace Violet proliferation assay.

SJL/PLP7 cells, which were modified with maleimide-, carboxylic acid-, biotin-, and WGA-functionalized nanoparticles at an initial nanoparticle/cell ratio of 5000, to the adhesion protein ICAM-1 was studied. ICAM-1 was selected as it has been identified as a critical adhesion molecule that mediates $\mathrm{T}$ cell polarization and crawling in the extravasation of $\mathrm{CD}^{+} \mathrm{T}_{\mathrm{EM}}$ cells across the $\mathrm{BBB} \cdot{ }^{55,64} \mathrm{~T}$ cell binding to ICAM-1 was assessed by immobilizing the protein on a glass substrate and comparing the number of adhered cells. As a control experiment, to validate that binding is ICAM-1specific, cells were also presented to substrates that were modified with delta and notch-like epidermal growth factorrelated receptor (DNER), which is a protein that is not involved in the $\mathrm{BBB}$ diapedesis of $\mathrm{CD}^{+} \mathrm{T}_{\mathrm{EM}}$ cells. The ICAM1 binding properties of the nanoparticle-decorated cells are summarized in Figure 8. The data in Figure 8 show that ICAM-1 binding is not impaired by the presence of the nanoparticle payload on the cell surface irrespective of the immobilization chemistry used. Some cells, most notably those that were modified with biotin or WGA-functionalized nanoparticles, even show an increased ICAM-1 binding compared to the unmodified control $\mathrm{T}$ cells. All $\mathrm{T}$ cells, both unmodified control and nanoparticle-decorated cells, show negligible binding to DNER-coated substrates, illustrating that binding of the cells to ICAM-1 is specific.

The BBB migratory behavior of the nanoparticle-modified cells was further studied in vitro with a transendothelial migration (TEM assay) (Figure 9A). The TEM assay investigates migration of the $\mathrm{T}$ cells under static conditions across a monolayer of primary mouse brain microvascular endothelial ( $\mathrm{pMBMEC}$ ) cells that have been grown onto a porous filter membrane. This model was used because it retains some $\mathrm{BBB}$ characteristics such as complex tight junctions and low permeability. ${ }^{51}$ To mimic inflammation and promote transmigration, pMBMECs were stimulated with interleukin $1 \beta 16 \mathrm{~h}$ prior to the experiment. These experiments were carried out with SJL/PLP7 cells that were modified with maleimide-, carboxylic acid-, biotin-, and WGAfunctionalized nanoparticles at a nanoparticle/cell ratio of

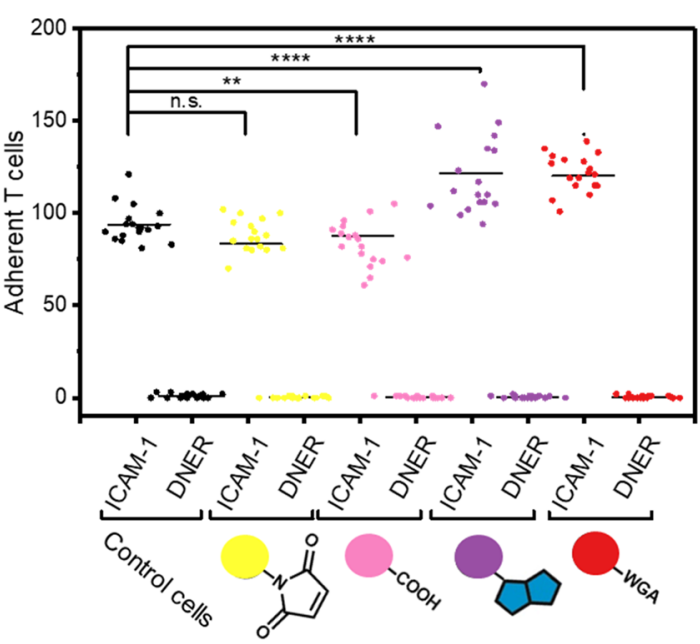

Figure 8. SJL/PLP7 T-Cell binding to ICAM-1. T Cell count of a binding assay on ICAM-1-coated wells and DNER-coated wells for unmodified control cells and nanoparticle-decorated T cells (prepared at an initial nanoparticle/cell ratio of 5000 nanoparticles/cell) immobilized with different chemistries performed at room temperature for $30 \mathrm{~min}$ under moderate shear conditions. Each dot represents one cell count from the diagonal of a $10 \mathrm{~nm} \times 10 \mathrm{~nm} /$ 10 divisions counting reticle using a $20 \mathrm{x}$ objective. The figure represents the results of two independent assays performed in triplicate. Each well was counted at three different positions. The horizontal bar represents the mean over all counts. $P$-values were determined by a $t$-test: carboxylic acid-functionalized nanoparticles: $P$ $<0.01$, maleimide-functionalized nanoparticles: $P>0.05$, WGAfunctionalized nanoparticles: $P<0.001$, and biotin-functionalized nanoparticles: $P<0.001$.

5000. Prior to the conjugation of the maleimide-functionalized nanoparticles, the cells were pretreated with TCEP. As a control, nonmodified SJL/PLP7 cells were used. Figure 9B shows the percentage of cells that have migrated across the pMBMEC monolayer after $6-8 \mathrm{~h}$ relative to the input as determined by flow cytometry. In this figure, each symbol represents a single condition of a set of triplicates. The data 
A

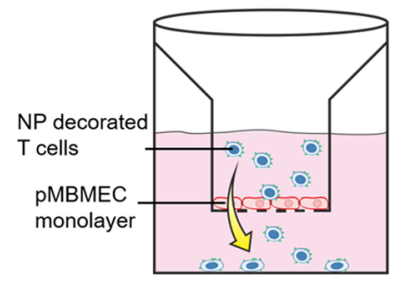

\section{B}

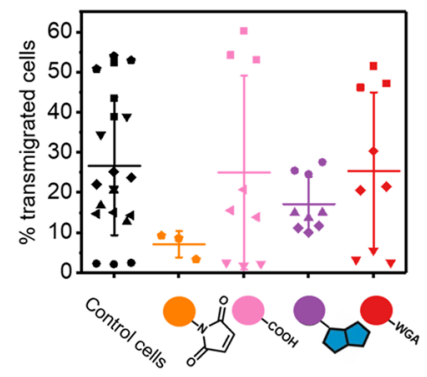

Figure 9. (A) Schematic illustration of the transendothelial migration assay (TEM) setup. The cell layer is made of stimulated primary mouse brain microvascular endothelial cells (pMBMECs) on porous filter inserts. A total of 100000 cells decorated with nanoparticles (nanoparticle/cell ratio of 5000) were added for a transmigration time of $6 \mathrm{~h}$. (B) Percentages of migrated T cells as determined by flow cytometry for control cells and cells modified with biotin-, WGA-, carboxylic acid-, or maleimide-functionalized nanoparticles. All data points represent a transmigration time of $6 \mathrm{~h}$, except the black squares, which are results from an experiment in which $8 \mathrm{~h}$ was chosen as transmigration time. The plot presents the results of three independent experiments performed in triplicate, and the error bars are standard deviations.

show that the percentage transmigrated cells within one experiment is relatively consistent, but can vary between different experiments. Overall, the migratory behavior of SJL/ PLP7 cells decorated with carboxylic acid-, biotin-, or WGAfunctionalized nanoparticles was not significantly affected by the surface conjugation of the nanoparticle payload. For SJL/ PLP7 cells that were pretreated with TCEP and subsequently decorated with maleimide-functionalized nanoparticles, a reduction in the number of migratory cells compared to the nonmodified control cells was observed, which was however not significant compared to the other nanoparticle-decorated cells and control cells.

In addition to quantifying the total number of cells that migrate across the pMBMEC monolayer, flow cytometry analysis also allows to determine the fraction of transmigrated cells that carry a nanoparticle payload and to assess a possible loss of nanoparticle cargo. For the biotin-, WGA-, carboxylic acid-, and maleimide nanoparticle-functionalized cells that were studied in the TEM experiments, Supporting Information Figure S11 compares the nanoparticle-associated fluorescence of the transmigrated cells with that of the input cells as well as unmodified control $\mathrm{T}$ cells. The flow cytometry histograms illustrate that $\mathrm{T}$ cells, which were modified with maleimide-, carboxylic acid-, or WGA-functionalized nanoparticles, lose a significant fraction of the nanoparticles during diapedesis as indicated by the partial overlap of the flow cytometry histograms of the nanoparticle-modified cells with that of the control cells. In contrast, cells that were modified with biotinfunctionalized nanoparticles lost fewer nanoparticles upon transmigration. Figure 10 presents a quantitative summary of these flow cytometry analyses and shows for each of the different nanoparticle conjugation chemistries, the percentage of transmigrated cells that carry a nanoparticle payload (Figure $10 \mathrm{~A})$ as well as the nanoparticle-associated mean fluorescence intensity of the transmigrated cells carrying nanoparticles relative to that of the input cells (Figure 10B). The latter is used as a measure to express the extent of nanoparticle loss. Figure $10 \mathrm{~A}$ reveals that $61 \pm 7 \%$ of all transmigrated,

A
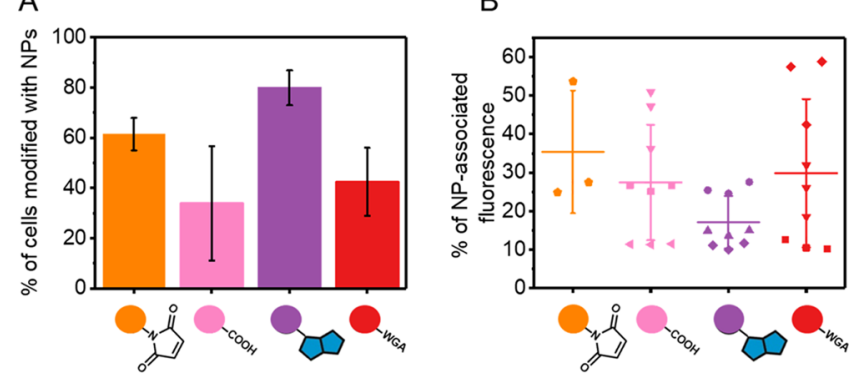

Figure 10. (A) Percentage of $\mathrm{T}$ cells after migration across the pMBMEC monolayer, which still carries nanoparticles (DiOpositive). (B) Percentage of nanoparticle-associated fluorescence of the subpopulation of $\mathrm{T}$ cells, which is still carrying nanoparticles compared to the nanoparticle-modified cells that were used as input in the transendothelial migration assay. The histogram presents the results of three independent experiments performed in triplicate, and the error bars are standard deviations. The results obtained with the maleimide nanoparticles were performed as one experiment in triplicate.

maleimide nanoparticle-modified cells, $34 \pm 23 \%$ of the transmigrated, carboxylic acid-functionalized nanoparticlemodified cells, $80 \pm 7 \%$ of the transmigrated, biotin nanoparticle-modified cells, and $43 \pm 14 \%$ of all transmigrated, WGA-functionalized nanoparticle-modified cells still carry nanoparticles. To evaluate the number of nanoparticles on the surface of the transmigrated cells compared to the surface concentration of nanoparticles on the input cells, the mean fluorescence intensity of the subpopulation which carries nanoparticles was compared with the mean fluorescent intensity of all of the cells directly after the nanoparticle modification at $t=0$ (Figure 10B). This analysis reveals that $\mathrm{T}$ cells that managed to migrate across this model, in vitro $\mathrm{BBB}$ and that still contained a nanoparticle payload on average carried less than $50 \%$ of their initial payload. The loss of nanoparticles was least pronounced when cells were modified with maleimide-functionalized nanoparticles and most prominent when biotin-functionalized nanoparticles were bound to cell surface NeutrAvidin moieties.

Figure 11 shows fluorescent images taken from the luminal side of the pMBMEC monolayer after the transmigration assay with 100000 modified cells. Figure $11 \mathrm{~A}$ presents the pMBMEC monolayer after a transmigration experiment with Neutravidin-modified SJL/PLP7 cells carrying biotin-functionalized nanoparticles, and Figure $11 \mathrm{~B}$ is an image of the pMBMEC monolayer after an experiment with SJL/PLP7 cells covalently modified with maleimide-functionalized nanoparticles (for both experiments, SJL/PLP7 cells were used that were modified at an initial nanoparticle-cell ratio of 5000). The actin filaments of the fixed pMBMEC monolayer were stained with Phalloidin Rhodamine (Figure 11, red), and the pMBMEC nuclei were stained with DAPI (Figure 11, blue). To identify the $\mathrm{T}$ cells, first, a biotin moiety introduced through receptor-specific interactions (CD45) and subsequently a Cy5-conjugated streptavidin was immobilized (Figure 11, white). The fluorescent image in Figure 11A reveals the green fluorescence due to (clusters) of nanoparticles that have been lost by the cells during transmigration. The free nanoparticles and nanoparticle clusters on the cell monolayer confirm the above-observed behavior that the $\mathrm{T}$ cells lose nanoparticles during the transmigration (Figure 11A). However, also polarized $\mathrm{T}$ cells, which are currently 
A

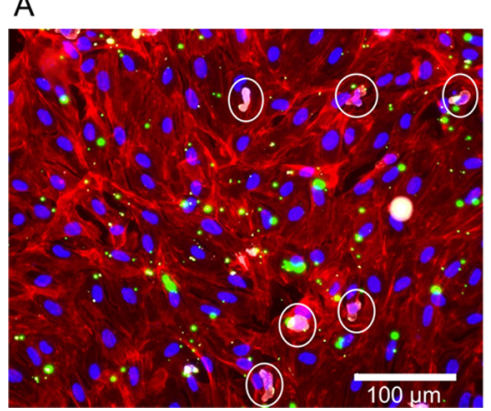

B

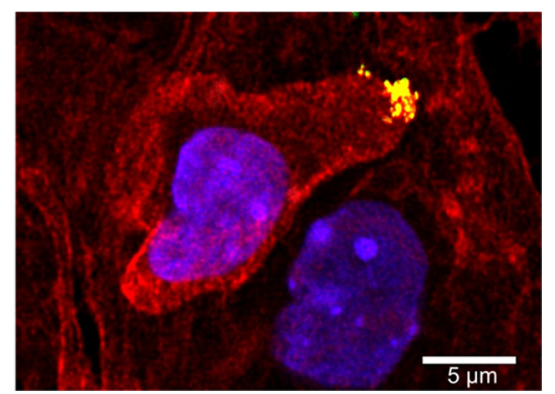

Figure 11. Fluorescent images of the pMBMEC monolayer after the transmigration assay. The actin filaments are shown in red (Rhodamine Phalloidin), the cell nuclei in blue (DAPI), biotin nanoparticles in green (DiO), and T cells in white (CD45 biotin, SA-Cy5). T cells carrying nanoparticles are highlighted with white circles. (A) Wide-field fluorescent image (20× magnification) of the luminal pMBMEC monolayer after a transmigration experiment with NeutrAvidin-modified cells carrying biotin-functionalized PLA nanoparticles (prepared at a nanoparticle/cell ratio of 5000). The white circles highlight nanoparticle-carrying SJL/PLP7 cells. (B) Confocal fluorescent image (63× magnification) of a maleimidefunctionalized PLA nanoparticle-modified, polarized SJL/PLP7 T cell (prepared at a nanoparticle/cell ratio of 5000), which is on the pMBMEC monolayer and presents its nanoparticle payload at the uropod.

undergoing the process of diapedesis, are visible. Activated $\mathrm{T}$ cells can be recognized by their elongated shape due to the morphological remodeling due to the interaction of the $\mathrm{T}$ cells and its ligands on the endothelial cell monolayer. ${ }^{65}$ This process leads to the rearrangement of the nanoparticles to the uropod (Figure 11B).

\section{CONCLUSIONS}

This study has investigated and compared the surface modification of two $\mathrm{T}$ cell lines with PLA nanoparticles using a range of cell surface conjugation chemistries. The $\mathrm{T}$ cell lines used in this study are human Jurkat $\mathrm{T}$ cells, as well as activated effector/memory $\mathrm{CD}^{+}$helper SJL/PLP7 $\mathrm{T}$ cells. These latter cells were chosen since they possess BBB migratory properties and are attractive candidates for the development of cell-based drug delivery systems to the CNS. A total of seven cell surface immobilization chemistries was explored, which included three covalent strategies (active ester-amine coupling, strain-promoted azide-alkyne cycloaddition, and thiol-maleimide Michael addition) and four noncovalent approaches (lectin-carbohydrate binding, electrostatic interactions, as well as biotin-NeutrAvidin-mediated conjugation using covalently or noncovalently cell surfaceimmobilized biotin moieties).

Flow cytometry analysis revealed that the nanoparticle surface concentration can be tuned by varying the initial nanoparticle/cell ratio used in the cell surface modification experiments. It was found that, for a given nanoparticle/cell ratio, noncovalent nanoparticle coupling strategies generally resulted in higher nanoparticle cell surface concentrations compared to the use of covalent chemistries. From the covalent chemistries that were screened, coupling of active ester or alkyne-functionalized nanoparticles resulted in the lowest nanoparticle cell surface concentrations. With the exception of the conjugation of NeutrAvidin- or WGAfunctionalized nanoparticles, where viabilities were found to decrease to $\sim 50 \%$ at very high initial nanoparticle/cell ratios, most of the conjugation chemistries did not significantly affect the viability of the Jurkat cells. The SJL/PLP7 cells were found to be more sensitive toward the cell surface conjugation of maleimide-, carboxylic acid-, and NeutrAvidin-functionalized nanoparticles compared to the Jurkat cells. Confocal microscopy imaging and data analysis demonstrated that at an initial nanoparticle/cell ratio of 5000, Jurkat cells could be modified with 76-184 nanoparticles/cell when maleimide-, carboxylic acid-, biotin-, or WGA-functionalized PLA nanoparticles were used. SJL/PLP7 T cells modified with biotinfunctionalized nanoparticles at an initial nanoparticle/cell ratio of 5000 contained $153 \pm 52$ nanoparticles/cell, which compares well with the results obtained on Jurkat cells. Modification of Jurkat cells with maleimide-, carboxylic acid-, or WGA-functionalized nanoparticles at initial nanoparticle/ cell ratios of 500, 2500, and 5000 did not significantly change cell proliferation. For Jurkat cells pretreated with TCEP before conjugation of maleimide-functionalized nanoparticles, in contrast, a slight increase in proliferation was observed, while for cells modified with biotin-functionalized nanoparticles, proliferation was slightly decreased compared to the control cells. Surface modification of SJL/PLP7 cells with carboxylic acid, biotin-, and WGA-functionalized nanoparticles at an initial nanoparticle/cell ratio of 5000 did not affect cell proliferation. As a first probe of the $\mathrm{BBB}$ migratory properties of the nanoparticle-decorated SJL/PLP7 cells, their ability to bind to ICAM-1 was assessed. These experiments revealed that ICAM-1 binding is not impaired by the presence of the nanoparticle payload irrespective of the immobilization chemistry used. Finally, the migratory behavior of nanoparticle-modified SJL/PLP7 cells was studied in a twochamber, in vitro $\mathrm{BBB}$ model. The migratory behavior of SJL/PLP7 cells carrying carboxylic acid-, biotin-, or WGAfunctionalized nanoparticles was not significantly affected by the presence of the nanoparticle payload. In contrast, however, for SJL/PLP7 cells decorated with maleimide-functionalized nanoparticles, a reduction in the number of migratory cells compared to the nonmodified control cells was observed. Flow cytometry analysis further revealed that $\mathrm{T}$ cells that migrated across the in vitro $\mathrm{BBB}$ model and which still contained a nanoparticle payload, on average carried less than $50 \%$ of their initial payload. The loss of nanoparticles was least pronounced for cells that carried maleimide-functionalized nanoparticles and most prominent when biotin-functionalized nanoparticles were attached to cell surface NeutrAvidin moieties.

While there has been an increased interest to explore cells as carriers for the transport and delivery of nanomedicines, only relatively little is known about the chemistry that underlies the surface modification of cell-based carriers with nanoparticle cargo. Investigating and understanding the impact of different nanoparticle-cell surface conjugation chemistries on the 
viability and functional properties of the cells will provide guidelines to further improve the design of cell-based nanoparticle delivery systems. The results of this study present a first step in this direction and provide first guidelines for the surface modification of $\mathrm{T}$ cells, in particular in view of their possible use for drug delivery to the CNS.

\section{ASSOCIATED CONTENT}

\section{SI Supporting Information}

The Supporting Information is available free of charge at https://pubs.acs.org/doi/10.1021/acs.biomac.1c00488.

Experimental details: materials, procedures, nanoparticle synthesis and surface modification, cell surface modification, and quantification of functional groups on nanoparticles and cell surfaces; flow cytometry results; additional confocal microscopy results; and nanoparticle characterization data (PDF)

\section{AUTHOR INFORMATION}

\section{Corresponding Author}

Harm-Anton Klok - École Polytechnique Fédérale de Lausanne (EPFL), Institut des Matériaux and Institut des Sciences et Ingénierie Chimiques, Laboratoire des Polymères, CH-1015 Lausanne, Switzerland; 이이이.org/0000-00033365-6543; Email: harm-anton.klok@epfl.ch

\section{Authors}

Tanja Thomsen - Ecole Polytechnique Fédérale de Lausanne (EPFL), Institut des Matériaux and Institut des Sciences et Ingénierie Chimiques, Laboratoire des Polymères, $\mathrm{CH}-1015$ Lausanne, Switzerland

Regina Reissmann - University of Bern, Theodor Kocher Institute, CH-3000 Bern, Switzerland

Elisa Kaba - University of Bern, Theodor Kocher Institute, CH-3000 Bern, Switzerland

Britta Engelhardt - University of Bern, Theodor Kocher Institute, CH-3000 Bern, Switzerland

Complete contact information is available at:

https://pubs.acs.org/10.1021/acs.biomac.1c00488

\section{Notes}

The authors declare no competing financial interest.

\section{ACKNOWLEDGMENTS}

This work was financially supported by the Swiss National Science Foundation (SNSF). The authors are grateful to O. Burri and R. Guiet from the EPFL Biolmaging \& Optics Platform (BIOP) for their help with the image analysis.

\section{REFERENCES}

(1) Duncan, R. Polymer conjugates as anticancer nanomedicines. Nat. Rev. Cancer 2006, 6, 688-701.

(2) Davis, M. E.; Chen, Z. G.; Shin, D. M. Nanoparticle therapeutics: an emerging treatment modality for cancer. Nat. Rev. Drug Discovery 2008, 7, 771-782.

(3) Elsabahy, M.; Wooley, K. L. Design of polymeric nanoparticles for biomedical delivery applications. Chem. Soc. Rev. 2012, 41, 25452561.

(4) Couvreur, P. Nanoparticles in drug delivery: past, present and future. Adv. Drug Delivery Rev. 2013, 65, 21-23.

(5) Nicolas, J.; Mura, S.; Brambilla, D.; Mackiewicz, N.; Couvreur, P. Design, functionalization strategies and biomedical applications of targeted biodegradable/biocompatible polymer-based nanocarriers for drug delivery. Chem. Soc. Rev. 2013, 42, 1147-1235.

(6) Ferrari, R.; Sponchioni, M.; Morbidelli, M.; Moscatelli, D. Polymer nanoparticles for the intravenous delivery of anticancer drugs: the checkpoints on the road from the synthesis to clinical translation. Nanoscale 2018, 10, 22701-22719.

(7) Wilhelm, S.; Tavares, A. J.; Dai, Q.; Ohta, S.; Audet, J.; Dvorak, H. F.; Chan, W. C. W. Analysis of nanoparticle delivery to tumours. Nat. Rev. Mater. 2016, 1, No. 16014.

(8) Danhier, F. To exploit the tumor microenvironment: Since the EPR effect fails in the clinic, what is the future of nanomedicine? J. Controlled Release 2016, 244, 108-121.

(9) Stephan, M. T.; Irvine, D. J. Enhancing cell therapies from the outside in: Cell surface engineering using synthetic nanomaterials. Nano Today 2011, 6, 309-325.

(10) Yoo, J.-W.; Irvine, D. J.; Discher, D. E.; Mitragotri, S. Bioinspired, bioengineered and biomimetic drug delivery carriers. Nat. Rev. Drug Discovery 2011, 10, 521-535.

(11) Su, Y.; Xie, Z.; Kim, G. B.; Dong, C.; Yang, J. Design strategies and applications of circulating cell-mediated drug delivery systems. ACS Biomater. Sci. Eng. 2015, 1, 201-217.

(12) Fliervoet, L. A. L.; Mastrobattista, E. Drug delivery with living cells. Adv. Drug Delivery Rev. 2016, 106, 63-72.

(13) Villa, C. H.; Anselmo, A. C.; Mitragotri, S.; Muzykantov, V. Red blood cells: Supercarriers for drugs, biologicals, and nanoparticles and inspiration for advanced delivery systems. Adv. Drug Delivery Rev. 2016, 106, 88-103.

(14) Ayer, M.; Klok, H. A. Cell-mediated delivery of synthetic nanoand microparticles. J. Controlled Release 2017, 259, 92-104.

(15) Singh, B.; Mitragotri, S. Harnessing cells to deliver nanoparticle drugs to treat cancer. Biotechnol. Adv. 2020, 42, No. 107339.

(16) Banskota, S.; Yousefpour, P.; Chilkoti, A. Cell-Based Biohybrid Drug Delivery Systems: The Best of the Synthetic and Natural Worlds. Macromol. Biosci. 2017, 17, No. 1600361.

(17) Pang, L.; Zhang, C.; Qin, J.; Han, L.; Li, R.; Hong, C.; He, H.; Wang, J. A novel strategy to achieve effective drug delivery: exploit cells as carrier combined with nanoparticles. Drug Delivery 2017, 24, $83-91$.

(18) Zhao, Z.; Ukidve, A.; Kim, J.; Mitragotri, S. Targeting Strategies for Tissue-Specific Drug Delivery. Cell 2020, 181, 151-167.

(19) Agrahari, V.; Agrahari, V.; Mitra, A. K. Next generation drug delivery: circulatory cells-mediated nanotherapeutic approaches. Expert Opin. Drug Delivery 2017, 14, 285-289.

(20) Thomsen, T.; Klok, H.-A. Chemical Cell Surface Modification and Analysis of Nanoparticle-Modified Living Cells. ACS Appl. Bio Mater. 2021, 4, 2293-2306.

(21) Chambers, E.; Mitragotri, S. Long Circulating Nanoparticles via Adhesion on Red Blood Cells: Mechanism and Extended Circulation. Exp. Biol. Med. 2007, 232, 958-966.

(22) Chambers, E.; Mitragotri, S. Prolonged circulation of large polymeric nanoparticles by non-covalent adsorption on erythrocytes. J. Controlled Release 2004, 100, 111-119.

(23) Anselmo, A. C.; Gupta, V.; Zern, B. J.; Pan, D.; Zakrewsky, M.; Muzykantov, V.; Mitragotri, S. Delivering Nanoparticles to Lungs while Avoiding Liver and Spleen through Adsorption on Red Blood Cells. ACS Nano 2013, 7, 11129-11137.

(24) Brenner, J. S.; Pan, D. C.; Myerson, J. W.; Marcos-Contreras, O. A.; Villa, C. H.; Patel, P.; Hekierski, H.; Chatterjee, S.; Tao, J.-Q.; Parhiz, H.; Bhamidipati, K.; Uhler, T. G.; Hood, E. D.; Kiseleva, R. Y.; Shuvaev, V. S.; Shuvaeva, T.; Khoshnejad, M.; Johnston, I.; Gregory, J. V.; Lahann, J.; Wang, T.; Cantu, E.; Armstead, W. M.; Mitragotri, S.; Muzykantov, V. Red blood cell-hitchhiking boosts delivery of nanocarriers to chosen organs by orders of magnitude. Nat. Commun. 2018, 9, No. 2684.

(25) Zelepukin, I. V.; Yaremenko, A. V.; Shipunova, V. O.; Babenyshev, A. V.; Balalaeva, I. V.; Nikitin, P. I.; Deyev, S. M.; Nikitin, M. P. Nanoparticle-based drug delivery via RBC-hitchhiking for the inhibition of lung metastases growth. Nanoscale 2019, 11, $1636-1646$. 
(26) Wayteck, L.; Dewitte, H.; De Backer, L.; Breckpot, K.; Demeester, J.; De Smedt, S. C.; Raemdonck, K. Hitchhiking nanoparticles: Reversible coupling of lipid-based nanoparticles to cytotoxic T lymphocytes. Biomaterials 2016, 77, 243-254.

(27) Stephan, M. T.; Moon, J. J.; Um, S. H.; Bershteyn, A.; Irvine, D. J. Therapeutic cell engineering with surface-conjugated synthetic nanoparticles. Nat. Med. 2010, 16, 1035-1041.

(28) Stephan, M. T.; Stephan, S. B.; Bak, P.; Chen, J.; Irvine, D. J. Synapse-directed delivery of immunomodulators using T-cellconjugated nanoparticles. Biomaterials 2012, 33, 5776-5787.

(29) Timin, A. S.; Litvak, M. M.; Gorin, D. A.; Atochina-Vasserman, E. N.; Atochin, D. N.; Sukhorukov, G. B. Cell-Based Drug Delivery and Use of Nano-and Microcarriers for Cell Functionalization. Adv. Healthcare Mater. 2018, 7, No. 1700818.

(30) Park, J.; Andrade, B.; Seo, Y.; Kim, M.-J.; Zimmerman, S. C.; Kong, H. Engineering the Surface of Therapeutic "Living" Cells. Chem. Rev. 2018, 118, 1664-1690.

(31) Abbina, S.; Siren, E. M. J.; Moon, H.; Kizhakkedathu, J. N. Surface Engineering for Cell-Based Therapies: Techniques for Manipulating Mammalian Cell Surfaces. ACS Biomater. Sci. Eng. 2018, 4, 3658-3677.

(32) Wibroe, P. P.; Anselmo, A. C.; Nilsson, P. H.; Sarode, A.; Gupta, V.; Urbanics, R.; Szebeni, J.; Hunter, A. C.; Mitragotri, S.; Mollnes, T. E.; Moghimi, S. M. Bypassing adverse injection reactions to nanoparticles through shape modification and attachment to erythrocytes. Nat. Nanotechnol. 2017, 12, 589-594.

(33) Armstrong, J. P. K.; Shakur, R.; Horne, J. P.; Dickinson, S. C.; Armstrong, C. T.; Lau, K.; Kadiwala, J.; Lowe, R.; Seddon, A.; Mann, S.; Anderson, J. L. R.; Perriman, A. W.; Hollander, A. P. Artificial membrane-binding proteins stimulate oxygenation of stem cells during engineering of large cartilage tissue. Nat. Commun. 2015, 6, No. 7405 .

(34) Emmetiere, F.; Irwin, C.; Viola-Villegas, N. T.; Longo, V.; Cheal, S. M.; Zanzonico, P.; Pillarsetty, N.; Weber, W. A.; Lewis, J. S.; Reiner, T. 18F-Labeled-Bioorthogonal Liposomes for In Vivo Targeting. Bioconjugate Chem. 2013, 24, 1784-1789.

(35) Cho, J.; Kushiro, K.; Teramura, Y.; Takai, M. Lectin-Tagged Fluorescent Polymeric Nanoparticles for Targeting of Sialic Acid on Living Cells. Biomacromolecules 2014, 15, 2012-2018.

(36) Jacobs, J.; Byrne, A.; Gathergood, N.; Keyes, T. E.; Heuts, J. P. A.; Heise, A. Facile Synthesis of Fluorescent Latex Nanoparticles with Selective Binding Properties Using Amphiphilic Glycosylated Polypeptide Surfactants. Macromolecules 2014, 47, 7303-7310.

(37) Xu, M.; Asghar, S.; Dai, S.; Wang, Y.; Feng, S.; Jin, L.; Shao, F.; Xiao, Y. Mesenchymal stem cells-curcumin loaded chitosan nanoparticles hybrid vectors for tumor-tropic therapy. Int. J. Biol. Macromol. 2019, 134, 1002-1012.

(38) Cao, P.; Mooney, R.; Tirughana, R.; Abidi, W.; Aramburo, S.; Flores, L.; Gilchrist, M.; Nwokafor, U.; Haber, T.; Tiet, P.; Annala, A. J.; Han, E.; Dellinger, T.; Aboody, K. S.; Berlin, J. M. Intraperitoneal Administration of Neural Stem Cell-Nanoparticle Conjugates Targets Chemotherapy to Ovarian Tumors. Bioconjugate Chem. 2017, 28, 1767-1776.

(39) Zheng, Y.; Tang, L.; Mabardi, L.; Kumari, S.; Irvine, D. J. Enhancing Adoptive Cell Therapy of Cancer through Targeted Delivery of Small-Molecule Immunomodulators to Internalizing or Noninternalizing Receptors. ACS Nano 2017, 11, 3089-3100.

(40) Kolesnikova, T. A.; Kiragosyan, G.; Le, T. H. N.; Springer, S.; Winterhalter, M. Protein A Functionalized Polyelectrolyte Microcapsules as a Universal Platform for Enhanced Targeting of Cell Surface Receptors. ACS Appl. Mater. Interfaces 2017, 9, 11506-11517.

(41) Li, L.; Guan, Y.; Liu, H.; Hao, N.; Liu, T.; Meng, X.; Fu, C.; Li, Y.; Qu, Q.; Zhang, Y.; Ji, S.; Chen, L.; Chen, D.; Tang, F. Silica Nanorattle-Doxorubicin-Anchored Mesenchymal Stem Cells for Tumor-Tropic Therapy. ACS Nano 2011, 5, 7462-7470.

(42) Saxon, E.; Bertozzi, C. R. Cell Surface Engineering by a Modified Staudinger Reaction. Science 2000, 287, 2007-2010.

(43) Xie, Y.-Q.; Arik, H.; Wei, L.; Zheng, Y.; Suh, H.; Irvine, D. J.; Tang, L. Redox-responsive interleukin-2 nanogel specifically and safely promotes the proliferation and memory precursor differentiation of tumor-reactive T-cells. Biomater. Sci. 2019, 7, 1345-1357.

(44) Huang, B.; Abraham, W. D.; Zheng, Y.; Bustamante López, S. C.; Luo, S. S.; Irvine, D. J. Active targeting of chemotherapy to disseminated tumors using nanoparticle-carrying T cells. Sci. Transl. Med. 2015, 7, No. 291ra94.

(45) Siriwon, N.; Kim, Y. J.; Siegler, E.; Chen, X.; Rohrs, J. A.; Liu, Y.; Wang, P. CAR-T Cells Surface-Engineered with Drug-Encapsulated Nanoparticles Can Ameliorate Intratumoral T-cell Hypofunction. Cancer Immunol. Res. 2018, 6, 812-824.

(46) Wang, S.; Yin, D.; Wang, W.; Shen, X.; Zhu, J.-J.; Chen, H.-Y.; Liu, Z. Targeting and Imaging of Cancer Cells via MonosaccharideImprinted Fluorescent Nanoparticles. Sci. Rep. 2016, 6, No. 22757.

(47) Holden, C. A.; Yuan, Q.; Yeudall, W. A.; Lebman, D. A.; Yang, $\mathrm{H}$. Surface engineering of macrophages with nanoparticles to generate a cell-nanoparticle hybrid vehicle for hypoxia-targeted drug delivery. Int. J. Nanomed. 2010, 5, 25-36.

(48) Zhang, P.; Zhang, X.; Li, C.; Zhou, S.; Wu, W.; Jiang, X. TargetAmplified Drug Delivery of Polymer Micelles Bearing Staudinger Ligation. ACS Appl. Mater. Interfaces 2019, 11, 32697-32705.

(49) Lee, S.; Yoon, H. I.; Na, J. H.; Jeon, S.; Lim, S.; Koo, H.; Han, S.-S.; Kang, S.-W.; Park, S.-J.; Moon, S.-H.; Park, J. H.; Cho, Y. W.; Kim, B.-S.; Kim, S. K.; Lee, T.; Kim, D.; Lee, S.; Pomper, M. G.; Kwon, I. C.; Kim, K. In vivo stem cell tracking with imageable nanoparticles that bind bioorthogonal chemical receptors on the stem cell surface. Biomaterials 2017, 139, 12-29.

(50) Kamaly, N.; Yameen, B.; Wu, J.; Farokhzad, O. C. Degradable Controlled-Release Polymers and Polymeric Nanoparticles: Mechanisms of Controlling Drug Release. Chem. Rev. 2016, 116, 26022663.

(51) Ayer, M.; Schuster, M.; Gruber, I.; Blatti, C.; Kaba, E.; Enzmann, G.; Burri, O.; Guiet, R.; Seitz, A.; Engelhardt, B.; Klok, H.A. T Cell-Mediated Transport of Polymer Nanoparticles across the Blood-Brain Barrier. Adv. Healthcare Mater. 2021, 10, No. 2001375.

(52) Thomsen, T.; Ayoub, A. B.; Psaltis, D.; Klok, H.-A. Fluorescence-Based and Fluorescent Label-Free Characterization of Polymer Nanoparticle Decorated T Cells. Biomacromolecules 2021, 22, 190-200.

(53) Thomsen, T.; Klok, H.-A.; Burri, O.; Seitz, A. Imaris EasyXT Particle Distance Analysis (Matlab) (Version v1.0). Zenodo, Aug 25, 2020, DOI: 10.5281 /zenodo.3999185.

(54) Abadier, M.; Jahromi, N. H.; Alves, L. C.; Boscacci, R.; Vestweber, D.; Barnum, S.; Deutsch, U.; Engelhardt, B.; Lyck, R. Cell surface levels of endothelial ICAM-1 influence the transcellular or paracellular T-cell diapedesis across the blood-brain barrier. Eur. J. Immunol. 2015, 45, 1043-1058.

(55) Reiss, Y.; Hoch, G.; Deutsch, U.; Engelhardt, B. T cell interaction with ICAM-1-deficient endothelium in vitro: essential role for ICAM-1 and ICAM-2 in transendothelial migration of T cells. Eur. J. Immunol. 1998, 28, 3086-3099.

(56) Steiner, O.; Coisne, C.; Cecchelli, R.; Boscacci, R.; Deutsch, U.; Engelhardt, B.; Lyck, R. Differential roles for endothelial ICAM-1, ICAM-2, and VCAM-1 in shear-resistant T cell arrest, polarization, and directed crawling on blood-brain barrier endothelium. J. Immunol. 2010, 185, 4846-4855.

(57) Wilhelm, C.; Billotey, C.; Roger, J.; Pons, J. N.; Bacri, J. C.; Gazeau, F. Intracellular uptake of anionic superparamagnetic nanoparticles as a function of their surface coating. Biomaterials 2003, 24, 1001-1011.

(58) Nazarenus, M.; Zhang, Q.; Soliman, M. G.; Del Pino, P.; Pelaz, B.; Carregal-Romero, S.; Rejman, J.; Rothen-Rutishauser, B.; Clift, M. J. D.; Zellner, R.; Nienhaus, G. U.; Delehanty, J. B.; Medintz, I. L.; Parak, W. J. In vitro interaction of colloidal nanoparticles with mammalian cells: What have we learned thus far? Beilstein J. Nanotechnol. 2014, 5, 1477-1490.

(59) Verma, A.; Stellacci, F. Effect of Surface Properties on Nanoparticle-Cell Interactions. Small 2010, 6, 12-21. 
(60) Tohver, V.; Smay, J. E.; Braem, A.; Braun, P. V.; Lewis, J. A. Nanoparticle halos: A new colloid stabilization mechanism. Proc. Natl. Acad. Sci. U.S.A. 2001, 98, 8950-8954.

(61) McKee, C. T.; Walz, J. Y. Interaction forces between colloidal particles in a solution of like-charged, adsorbing nanoparticles. J. Colloid Interface Sci. 2012, 365, 72-80.

(62) Yang, B.; Gao, F.; Li, Z.; Li, M.; Chen, L.; Guan, Y.; Liu, G.; Yang, L. Selective Entropy Gain-Driven Adsorption of Nanospheres onto Spherical Bacteria Endows Photodynamic Treatment with Narrow-Spectrum Activity. J. Phys. Chem. Lett. 2020, 11, 2788-2796.

(63) Cha, J.; Kim, H.; Hwang, N. S.; Kim, P. Mild Reduction of the Cancer Cell Surface as an Anti-invasion Treatment. ACS Appl. Mater. Interfaces 2018, 10, 35676-35680.

(64) Steiner, O.; Coisne, C.; Engelhardt, B.; Lyck, R. Comparison of immortalized bEnd5 and primary mouse brain microvascular endothelial cells as in vitro blood-brain barrier models for the study of T cell extravasation. J. Cereb. Blood Flow Metab. 2011, 31, 315-327. (65) Sánchez-Madrid, F.; Serrador, J. M. Bringing up the rear: defining the roles of the uropod. Nat. Rev. Mol. Cell Biol. 2009, 10, $353-359$. 